\begin{tabular}{|c|l|}
\hline Title & Residues and topological invariants of singular holomorphic foliations1 \\
\hline Author(s) & Seade, J.; Suwa, T. \\
\hline Citation & Hokkaido University Preprint Series in Mathematics, 335, 1-28 \\
\hline Issue Date & 1996-4-1 \\
\hline DOI & 10.14943/83481 \\
\hline Doc URL & http://hdl.handle.net/2115/69085 \\
\hline Type & bulletin (article) \\
\hline File Information & pre335.pdf \\
\hline
\end{tabular}

Instructions for use 


\section{RESIDUES AND TOPOLOGICAL INVARIANTS \\ OF SINGULAR HOLOMORPHIC FOLIATIONS ${ }^{1}$}

J. Seade and T. Suwa

Series \#335. April 1996 


\section{HOKKAIDO UNIVERSITY \\ PREPRINT SERIES IN MATHEMATICS}

\#311 N. Kawazumi, On the stable cohomology algebra of extended mapping class groups for surfaces, 13 pages. 1995.

\#312 H.M.Ito and T. Mikami, Poissonian asymptotics of a randomly perturbed dynamical system: Flip-flop of the Stochastic Disk Dynamo, 20 pages. 1995.

\#313 T. Nakazi, Slice maps and multipliers of invariant subspaces, 11 pages. 1995.

\#314 T. Mikami, Weak convergence on the first exit time of randomly perturbed dynamical systems with a repulsive equilibrium point, 20 pages. 1995 .

\#315 A. Arai, Canonical commutation relations, the Weierstrass Zetafunction, and infinite dimensional Hilbert space representations of the quantum group $U_{q}\left(\mathfrak{s l}_{2}\right), 22$ pages. 1995.

\#316 Y. Shibukawa, Vertex-face correspondence in elliptic solutions of the Yang-Baxter equation, 8 pages. 1995.

\#317 M.-H. Giga and Y. Giga, Consistency in evolutions by crystalline curvature, 16 pages. 1995.

\#318 Wei-Zhi Sun, Shadows of moving surfaces, 19 pages. 1995.

\#319 S. Izumiya and G.T. Kossioris, Bifurcations of shock waves for viscosity solutions of Hamilton-Jacobi equations of one space variable, 39 pages. 1995.

\#320 T. Teruya, Normal intermediate subfactors, 44 pages. 1995.

\#321 M. Ohnuma, Axisymmetric solutions and singular parabolic equations in the theory of viscosity solutions, 26 pages. 1995.

\#322 T. Nakazi, An outer function and several important functions in two variables, 12 pages. 1995.

\#323 N. Kawazumi, An infinitesimal approach to the stable cohomology of the moduli of Riemann surfaces, 22 pages. 1995.

\#324 A. Arai, Factorization of self-adjoint operators by abstract Dirac operators and its application to second quantizations on Boson Fermion Fock spaces, 15 pages. 1995.

\#325 K. Sugano, On strongly separable Frobenius extensions, 11 pages. 1995.

\#326 D. Lehmann and T. Suwa, Residues of holomorphic vector fields on singular varieties, 21 pages. 1995.

\#327 K. Tsutaya, Local regularity of non-resonant nonlinear wave equations, 23 pages. 1996.

\#328 T. Ozawa and Y. Tsutsumi, Space-time estimates for null gauge forms and nonlinear Schrödinger equations, 25 pages. 1996.

\#329 O. Ogurisu, Anticommutativity and spin 1/2 Schrödinger operators with magnetic fields, 12 pages. 1996.

\#330 Y. Kurokawa, Singularities for projections of contour lines of surfaces onto planes, 24 pages. 1996.

\#331 M.-H. Giga and Y. Giga, Evolving graphs by singular weighted curvature, 94 pages. 1996.

\#332 M. Ohnuma and K. Sato, Singular degenerate prarabolic equations with applications to the $p$-laplace diffusion equation, 20 pages. 1996.

\#333 T. Nakazi, The spectra of Toeplitz operators with unimodular symbols, 9 pages. 1996.

\#334 B. Khanedani and T. Suwa, First variation of horomorphic forms and some applications, 11 pages. 1996. 


\title{
RESIDUES AND TOPOLOGICAL INVARIANTS OF SINGULAR HOLOMORPHIC FOLIATIONS ${ }^{1}$
}

\author{
José Seade and Tatsuo SuWa
}

The theorem of Poincaré-Hopf says that the total index of a vector field $v$ on a closed, smooth, oriented $n$-manifold $M$ is independent of the vector field and it equals the Euler-Poincare characteristic of $M, \chi(M)$. A vector field $v$ is actually determined, up to scaling, by the foliation on $M$ given by its integral curves. It is natural to ask whether there is a similar theorem for higher dimensional oriented foliations. If the foliation $\mathcal{F}$ is non-singular, this is well known: One has,

$$
\chi(M)=e(M)[M]=e(T \mathcal{F}) \cdot e(N \mathcal{F})[M],
$$

where $e()$ is the Euler class, $T \mathcal{F}$ is the tangent bundle of $\mathcal{F}, N \mathcal{F}$ is its normal bundle with respect to some riemannian metric, and $[M]$ is the orientation cycle. However, if the foliation $\mathcal{F}$ is singular, the question is more interesting. If the manifold $M$ and the foliation $\mathcal{F}$ are both complex analytic, then one has the tangent and the normal sheaves of $\mathcal{F}$. Both sheaves are coherent $[\mathrm{BB}]$, so they have resolutions by vector bundles, giving rise to well defined Chern classes of these sheaves [AH]. These classes satisfy:

$$
\begin{aligned}
\chi(M) & =c_{n}(M)[M]=\left(c_{n}(\mathcal{F})+c_{n-1}(\mathcal{F}) \cdot c_{1}(Q)+\cdots+c_{n}(Q)\right)[M] \\
& =c_{p}(\mathcal{F}) \cdot c_{n-p}(Q)[M]+[\text { Contribution of singular set } S]
\end{aligned}
$$

where $\mathcal{F}$ is the tangent sheaf, $Q$ is the normal sheaf, $p$ is the rank (or leaf dimension) of $\mathcal{F}$, and the "contribution of $S$ " involves all the terms that vanish when $\mathcal{F}$ is nonsingular. One has similar formulae for the lower Chern classes of $M$ in terms of those of $\mathcal{F}$ and $Q$. This "contribution of $S$ " is somehow explained by P. Baum and $R$. Bott in [BB]: They proved that given any homogeneous symmetric polynomial $\varphi$ of degree $d>n-p$, there exists a homology class $\operatorname{Res}_{\varphi}(\mathcal{F}, S) \in H_{2 n-2 d}(S ; \mathbb{C})$, where $S$ is the singular set of $\mathcal{F}$, such that

$$
\varphi(Q)=\mu_{*} \operatorname{Res}_{\varphi}(\mathcal{F}, S)
$$

\footnotetext{
${ }^{1}$ Research partially supported by CONACYT, Mexico and the Ministry of Education, Science and Culture, Japan, Grant-in aid for Scientific Research
} 
where $\mu_{*}$ is induced by the inclusion $S \hookrightarrow M$ followed by Poincaré duality. Generally speaking these residues are rather misterious and difficult to compute. However, in some cases they are well understood. For instance, it is well known (and we re-prove it here, in 4.3 below) that if $\mathcal{F}$ has complex dimension one, $\varphi$ is $\sigma_{n}$ (the elementary symmetric polynomial of degree $n$ ) and $S$ consists of isolated points $P_{1}, \ldots, P_{r}$, then there exists a vector field $v_{i}$ on a neighbourhood of each $P_{i}$, singular only at $P_{i}$ and tangent to $\mathcal{F}$, and $\operatorname{Res}_{\sigma_{n}}(\mathcal{F}, S)$ is the sum of the local indices of the $v_{i}$ 's. One has in this case:

$$
\chi(M)=c_{n}(M)[M]=c_{1}(\mathcal{F}) \cdot c_{n-1}(Q)[M]+\operatorname{Res}_{\sigma_{n}}(\mathcal{F}, S)
$$

If the codimension of $\mathcal{F}$ is one, there is a similar interpretation of the Baum-Bott residue [Su1].

This article can be regarded as being both, an extension of [BB] to open manifolds, and an extension of our previous article [SS] to higher dimensional foliations on singular varieties.

In $\S 1$ we extend the Baum-Bott theory of residues to singular holomorphic foliations on open manifolds which are relatively compact submanifolds of a complex manifold. We also discuss the behaviour of characteristic classes under the existence of non-singular vector fields, near the boundary, tangent to the foliation. In $\S 2$ and $\S 3$ we make the topological counterpart to $[\mathrm{BB}]$ and to $\S 1$; This applies to $C^{\infty}$, singular foliations on oriented manifolds. By comparing these two theories, the analytic one and the topological one, we obtain in $\S 4$ some new insights into the behaviour of the characteristic classes and the Baum-Bott residues of singular holomorphic foliations on complex manifolds. In $\S 5$ and $\S 6$ we study the case when the phase space is complex analytic, with isolated complete intersection singularities. We define, in $\S 6$, an invariant for foliations on germs of complete intersections with isolated singularity and prove that this is a topological invariant. This extends the index of 2-dimensional foliations defined in [GSV], and the Milnor number of a 1-dimensional holomorphic foliation defined in [CLS]. We also study its behaviour when we consider resolutions of the singularity.

The basic tool for defining this invariant is the index of a vector field on a singular variety, that we study in $\S 5$. There are several different notions of such index: The Schwartz index [Sc,BSc,KT], the GSV-index [Se,GSV,SS], the homological index of [G] and the differential index of [LSS], which turn out to (essentially) coincide. We determine the relationship between the Schwartz index and the GSVindex, and we prove a theorem about the total index of a vector field on a compact variety whose singularities are all isolated complete intersections (ICIS). As an application, we give a formula for the Chern number of the virtual tangent bundle of a (strong) local complete intersection with isolated singularities, which is a generalization of the classical adjunction formula for singular curves in surfaces. It is also used in showing a formula for the Chern-Schwartz-MacPherson class of such a variety in [Su2]. 
While working on this article, the first named author made a couple of visits to Hokkaido University in Sapporo, as well as to Ryukoku University in Kyoto, to IMPA at Rio de Janeiro and CIMAT in Guanajuato, Mexico. Also, the second named author visited CIMAT, UNAM in Mexico City, Université de Montpellier and CIRM in Marseille. We would like to thank these institutions for their support and hospitality. We are also grateful to J.-P. Brasselet, T. Ito and D. Lehmann for helpful conversations.

\section{§1. Chern classes and the Baum-Bott residues}

Let $M$ be a connected, relatively compact open set in a complex manifold of dimension $n>1$, with non-empty smooth boundary $\partial M$. For instance, $M$ can be taken to be a neighbourhood of a compact, connected component of the singular set of a holomorphic foliation on a complex manifold. We denote by $T M$ the holomorphic tangent bundle of $M$. Also, denoting by $\mathcal{O}_{M}$ the structure sheaf of $M$, let $\Theta_{M}=\mathcal{O}_{M}(T M)$ be the tangent sheaf of $M$. (The tangent sheaf of) a singular holomorphic foliation on $M$ is defined ([BB, p.281]) to be a full integrable coherent subsheaf $\mathcal{F}$ of $\Theta$. Set $Q=\Theta_{M} / \mathcal{F}$, the quotient sheaf; $Q$ is the normal sheaf of the foliation, and one has the exact sequence

$$
0 \rightarrow \mathcal{F} \rightarrow \Theta_{M} \rightarrow Q \rightarrow 0 .
$$

The singular set $S$ of $\mathcal{F}$ is the set of points where $Q$ is not $\mathcal{O}_{M}$-free. We assume that $S$ does not intersect a neighbourhood of the boundary of $M$ and that the codimension of $S$ is at least two. The sheaf $\mathcal{F}$ defines an ordinary foliation on $M-S$, and we let $p$ be the dimension of the leaves of this foliation. In what follows, we do not distinguish between a holomorphic vector bundle and the corresponding locally free sheaf. Thus away from $S, \mathcal{F}$ and $Q$ are vector bundles of ranks $p$ and $n-p$, respectively.

We recall $[\mathrm{BB}]$ that on $M-S, \mathcal{F}$ "acts" on $Q$, because $\mathcal{F}$ is integrable: If $\eta: \Theta \rightarrow Q$ is the projection, then the action is given by

$$
(u, \eta(v)) \mapsto[u, \eta(v)]=\eta([u, v]),
$$

for every $u \in \mathcal{F}$ and $\eta(v) \in Q$. Thus one has a partial connection for $Q$ on $M-S$, [BB, p.290],

$$
\delta: C^{\infty}(Q) \rightarrow C^{\infty}\left((\mathcal{F} \oplus \bar{T} M)^{*} \otimes Q\right) \simeq C^{\infty}\left(\operatorname{Hom}(\mathcal{F}, Q) \oplus \bar{T}^{*} M \otimes Q\right),
$$

defined by $\delta(s)=(u \mapsto[u, s], \bar{\partial} s)$. One can easily see [BB; 2.5] that there exists a connection $D_{-1}$ for $Q$ on $M-S$ extending $\delta$, i.e., the following diagram is commutative:

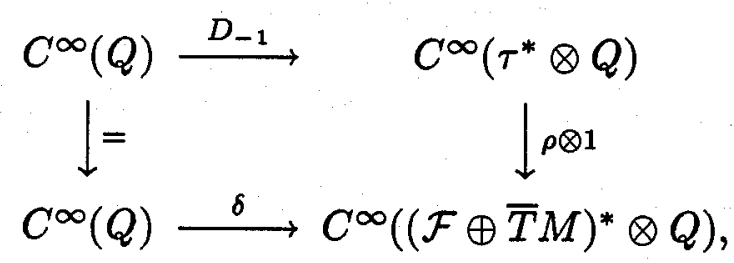


where $\tau=T M \oplus \bar{T} M$ and $\rho$ is the canonical surjection $\tau^{*} \rightarrow(\mathcal{F} \oplus \bar{T} M)^{*}$. Any connection on $Q$ extending the partial connection is called a basic connection ([BB]).

Since $Q$ is coherent, we can take a resolution by vector bundles on $M([\mathrm{AH}])$

$$
0 \rightarrow L_{r} \rightarrow \cdots \rightarrow L_{0} \rightarrow Q \rightarrow 0
$$

and connections $\left(D_{r}^{\prime}, \ldots, D_{0}^{\prime}, D_{-1}\right)$ on $M-S$, compatible with this sequence, where $D_{-1}$ is a basic connection for $Q$ on $M-S,[\mathrm{BB} ; 4.17]$. Let $\left\{Z_{\alpha}\right\}$ be the connected components of $S$. For each $Z_{\alpha}$, we take a regular neighbourhood $U_{\alpha}$ of $Z_{\alpha}$ and a compact set $\Sigma_{\alpha}$ so that $U_{\alpha} \cap U_{\beta}=\phi$ if $\alpha \neq \beta$ and that $Z_{\alpha} \subset$ Int $\Sigma_{\alpha} \subset \Sigma_{\alpha} \subset U_{\alpha}$. Set $\Sigma=\cup \Sigma_{\alpha}$, and extend each $D_{i}^{\prime}, 0 \leq i \leq r$, to a connection $D_{i}$ for $L_{i}$ on $M$ which coincides with $D_{i}^{\prime}$ on $M-\Sigma$, , BB;4.41]. The connections $D_{i}$ define curvatures $K_{i}$ on $M$ and $D_{-1}$ defines $K_{-1}$ on $M-S$. Since they are compatible with the exact sequence on $M-\Sigma$, we have

$$
\left(1+\sigma_{1}\left(K_{-1}\right)+\cdots+\sigma_{n}\left(K_{-1}\right)\right)=\prod_{j=0}^{r}\left(1+\sigma_{1}\left(K_{j}\right)+\cdots+\sigma_{n}\left(K_{j}\right)\right)^{\varepsilon(j)}
$$

where $\sigma_{1}, \ldots, \sigma_{n}$ are the elementary symmetric functions in $n$ variables and $\varepsilon(j)=$ $(-1)^{j}$. Define, for each $i=1, \ldots, n$, a $2 i$-form $\omega_{i}$ on $M$ by

$$
1+\omega_{1}+\cdots+\omega_{n}=\prod_{j=0}^{r}\left(1+\sigma_{1}\left(K_{j}\right)+\cdots+\sigma_{n}\left(K_{j}\right)\right)^{\varepsilon(j)}
$$

On $M-\Sigma, \omega_{i}=\sigma_{i}\left(K_{-1}\right)$, so these are 0 for $i>n-p$.

Let $\varphi$ be a homogeneous symmetric polynomial of degree $d$, and write it as a polynomial in the elementary symmetric functions:

$$
\varphi=P\left(\sigma_{1}, \ldots, \sigma_{n}\right),
$$

so by definition one has $\varphi(\omega)=P\left(\omega_{1}, \ldots, \omega_{n}\right)$, a closed $2 d$-form on $M$. One has,

$$
\varphi(\omega)=P\left(\sigma_{1}\left(K_{-1}\right), \ldots, \sigma_{n}\left(K_{-1}\right)\right)
$$

on $M-\Sigma$. If $d>n-p$, this form is identically 0 , by Bott's vanishing theorem [BB; 3.27], because $D_{-1}$ is basic. Hence $\varphi(\omega)$ has support in $\Sigma$. Therefore $\left(\frac{\sqrt{-1}}{2 \pi}\right)^{d} \varphi(\omega)$ represents a relative class

$$
\tilde{\varphi}(Q) \in H^{2 d}(M, M-S ; \mathbb{C}),
$$

whose image $j^{*}(\tilde{\varphi}(Q))$ is $\varphi(Q)$ in $H^{2 d}(M ; \mathbb{C})$. By Alexander-Lefschetz duality one has

$$
H^{2 d}(M, M-S ; \mathbb{C}) \underset{L}{\stackrel{\sim}{\longrightarrow}} H_{2 n-2 d}(S ; \mathbb{C})=\oplus_{\alpha} H_{2 n-2 d}\left(Z_{\alpha} ; \mathbb{C}\right) .
$$


In other words, we can write

$$
\tilde{\varphi}(Q)=\sum_{\alpha} \mu_{*} \operatorname{Res}_{\varphi}\left(\mathcal{F}, Z_{\alpha}\right)
$$

where $\mu_{*}=L^{-1}$ and $\operatorname{Res}_{\varphi}\left(\mathcal{F}, Z_{\alpha}\right)$ is a class in $H_{2 n-2 d}\left(Z_{\alpha} ; \mathbb{C}\right)$. Moreover, by $[\mathrm{BB}, 5.31]$, the class $\tilde{\varphi}(Q)$ does not depend on the choice of the basic connection nor the resolution of $Q$. Thus one has the following residue formula in the relative cohomology, which is basically in [BB].

1.1 Theorem. Let $\varphi$ be a homogeneous symmetric polynomial of degree $d>n-p$. Then, there exists a relative cohomology class, $\tilde{\varphi}(Q) \in H^{2 d}(M, \partial M ; \mathbb{C})$ that maps to $\varphi(Q) \in H^{2 d}(M ; \mathbb{C})$ under the morphism $j^{*}$ induced by the inclusion, and $\tilde{\varphi}(Q)$ is a sum of residues localized at the singular set $S$ of the foliation,

$$
\tilde{\varphi}(Q)=\sum_{\alpha} \mu_{*} \operatorname{Res}_{\varphi}\left(\mathcal{F}, Z_{\alpha}\right)
$$

where the sum runs over the connected components of $S$.

Note that in view of the commutative diagram

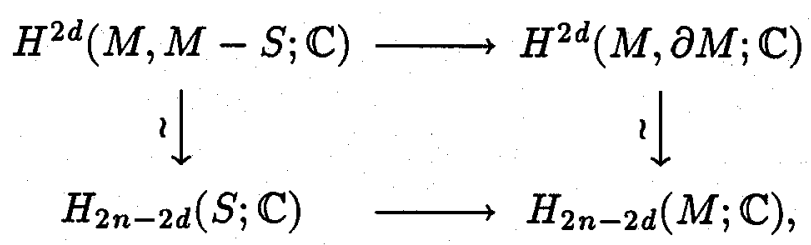

the formula $\left(1_{2}\right)$ is "more precise" than the one in the theorem.

Let us assume now that one has $C^{\infty}$ vector fields $s_{1}, \ldots, s_{k}$ on a neighbourhood $U$ of the boundary $\partial M$, linearly independent everywhere on $U$ and tangent to $\mathcal{F}, 0<k \leq p$. We refer to the set $\mathbf{f}=\left\{s_{1}, \ldots, s_{k}\right\}$ as a $k$-frame on $U$. We will use this $k$-frame to construct representatives of the Chern classes of $\mathcal{F}$ that vanish near $\partial M$. We let $F=\left.\mathcal{F}\right|_{U}$, and we let $F_{0}$ be the sub-bundle of $F$ spanned by $s_{1}, \ldots, s_{k}$. Thus on $U$,

$$
F=F_{0} \oplus F_{1},
$$

as $C^{\infty}$-bundles, where $F_{1}$ is the orthogonal complement of $F_{0}$. Let $\nabla_{0}$ be the trivial connection for $F_{0}$ determined by $\mathrm{f}$, and $\nabla_{1}$ an arbitrary connection for $F_{1}$. We define a connection $D^{\prime}$ for $F$ on $U$ by $D^{\prime}=\nabla_{0} \oplus \nabla_{1}$. We may take a compact set $C$ in $M$ such that $\overline{M-U}$ in contained in the interior of $C$, and a connection $D$ for $\mathcal{F}$ on $M-S$ such that $D=D^{\prime}$ on $M-C,[\mathrm{BB}, 4.41]$.

Taking a resolution of $\mathcal{F}$ by vector bundles on $M$, one has an exact sequence

$$
0 \rightarrow E_{q} \rightarrow \cdots \rightarrow E_{0} \rightarrow \mathcal{F} \rightarrow 0
$$


Let $\left(D_{q}^{\prime}, \ldots, D_{0}^{\prime}\right)$ be connections for the $E_{i}$ 's on $M-S$, such that $\left(D_{q}^{\prime}, \ldots, D_{0}^{\prime}, D\right)$ are compatible with the exact sequence. We extend each $D_{i}^{\prime}$ to a connection $D_{i}$ on $M$, which agrees with $D_{i}^{\prime}$ on $M-\Sigma$. We have curvature matrices $K_{q}, \ldots, K_{0}$, determined by $D_{q}, \ldots, D_{0}$. Define, for each $i=1, \ldots, n$, a (closed) $2 i$-form $\rho_{i}$ on $M$ by

$$
1+\rho_{1}+\cdots+\rho_{n}=\prod_{j=0}^{q}\left(1+\sigma_{1}\left(K_{j}\right)+\cdots+\sigma_{n}\left(K_{j}\right)\right)^{\varepsilon(j)} .
$$

Since $\left(D_{q}, \ldots, D_{0}, D\right)$ are compatible with the exact sequence on $M-\Sigma$, one has $\rho_{i}=\sigma_{i}(K)$ on $M-\Sigma$, where $K$ is the curvature matrix $K=d \theta-\theta \wedge \theta$ of the connection $D$. Also, since the connection matrix $\theta$ of $D$ (with respect to an appropriate frame) is of the form $\theta=\left(\begin{array}{cc}0 & 0 \\ 0 & \theta_{1}\end{array}\right)$ on $U-C$, with $\theta_{1}$ the connection matrix of $\nabla_{1}$, we have the following lemma.

1.2 Lemma. For $p-k<i \leq n$, one has $\sigma_{i}(K) \equiv 0$, on $U-C$.

The lemma above implies that the $\rho_{i}$ 's are globally defined and have compact support, in the appropiate range, since they vanish on $U-C$. Define

$$
\tilde{c}_{i}(\mathcal{F})=\left(\frac{\sqrt{-1}}{2 \pi}\right)^{i}\left[\rho_{i}\right] \in H^{2 i}(M, \partial M ; \mathbb{C}),
$$

for $i=p-k+1, \ldots, n$. These are the Chern classes of $\mathcal{F}$ relative to the $k$-frame f. By definition one has $j^{*}\left(\tilde{c}_{i}(\mathcal{F})\right)=c_{i}(\mathcal{F})$, so their image in $H^{*}(M ; \mathbb{C})$ under the homomorphism induced by the inclusion are the usual Chern classes of $\mathcal{F}$. We note however that the classes $\tilde{c}_{p+1}(\mathcal{F}), \ldots, \tilde{c}_{n}(\mathcal{F})$ actually vanish on $\partial M$ by rank reasons, because $\mathcal{F}$ is locally free of rank $p$ on a neighbourhood of $\partial M$, while $\tilde{c}_{p-k+1}(\mathcal{F}), \ldots, \tilde{c}_{p}(\mathcal{F})$ are relative because we have the $k$-frame $\mathbf{f}$, and they depend of the choice of $\mathbf{f}$ as relative classes. Hence we denote the latter classes by $c_{i}(\mathcal{F}, \mathbf{f})$ when we want to emphazise their dependence on $\mathbf{f}$.

Let $F_{0}$ be again the sub-vector bundle of $T M$ spanned by $\mathbf{f}$ on a neighbourhood $U^{\prime}$ of the boundary $\partial M$. Let $\nabla_{0}$ be the trivial connection for $F_{0}$ determined by $\mathbf{f}$ and $\hat{\nabla}_{1}$ some connection for the orthogonal complement of $F_{0}$ in $T M$. Then $\hat{D}^{\prime}=\nabla_{0} \oplus \hat{\nabla}_{1}$ is a connection for $T M$ on $U^{\prime}$. As before, we now extend $\hat{D}^{\prime}$ to a connection $\hat{D}$ for $T M$ on all of $M$, that coincides with $\hat{D}^{\prime}$ on a neighbourhood $U \subset U^{\prime}$ of $\partial M$. Let $\hat{K}$ be the curvature matrix of $\hat{D}$, so that the symmetric functions $\sigma_{i}(\hat{K})$ determine the usual Chern classes of $T M$,

$$
c_{i}(T M)=\left(\frac{\sqrt{-1}}{2 \pi}\right)^{i}\left[\sigma_{i}(\hat{K})\right] .
$$

We note that, as in Lemma $1.2, \sigma_{i}(\hat{K})$ vanishes identically on $U$ for $i>n-k$. Hence, these forms $\sigma_{i}(\hat{K})$ provide representatives $c_{i}(T M, \mathbf{f})$ of the Chern classes of $T M$, that vanish over $U$ for $i>n-k$, i.e., they are classes in $H^{2 i}(M, \partial M ; \mathbb{C})$. 
1.3 Definition. (cf. [Ke]) The Chern classes $c_{n-k+1}(T M, \mathbf{f}), \ldots, c_{n}(T M, \mathbf{f})$, are the Chern classes of $T M$ relative to the $k$-frame $\mathbf{f}$.

If $j^{*}$ is the inclusion homomorphism, then $j^{*}\left(c_{i}(T M, \mathbf{f})\right)$ is the usual Chern class $c_{i}(T M)$, but as a relative class $c_{i}(T M, \mathbf{f})$ does depend on the choice of the $k$-frame $\mathbf{f}$, generally speaking.

1.4 Theorem. Let $M$ and $\mathcal{F}$ be as above. Suppose we are given $k C^{\infty}$ sections $s_{1}, \ldots, s_{k}$ of $T M$ on a neighbourhood $U$ of $\partial M, 1 \leq k \leq p$, which are everywhere tangent to $\mathcal{F}$ and linearly independent over $\mathbb{C}$. Then, for all $j$ with $n-k+1 \leq j \leq n$, one has:

$$
c_{j}(T M, \mathbf{f})=\sum_{i=0}^{j-p+k-1} c_{i}(Q) \cdot \tilde{c}_{j-i}(\mathcal{F})+\sum_{i=j-p+k}^{j} \tilde{c}_{i}(Q) \cdot c_{j-i}(\mathcal{F})
$$

where $c_{i}(T M, \mathbf{f}), \tilde{c}_{i}(\mathcal{F}) \in H^{2 i}(M, \partial M)$, are the Chern classes of $M$ and $\mathcal{F}$, respectively, relative to the $k$-frame $\mathbf{f}=\left\{s_{1}, \ldots, s_{k}\right\}$, and the $\tilde{c}_{i}(Q)$ 's are the representatives of the Chern classes of the normal sheaf $Q$ defined above. Moreover, each $\tilde{c}_{i}(Q)$ is localized at the singular set $S$ of $\mathcal{F}$ : (Residue formula in relative cohomology)

$$
\tilde{c}_{i}(Q)=\sum_{Z \subset S} \mu_{*} \operatorname{Res}_{\sigma_{i}}(\mathcal{F}, Z), \quad n-p+1 \leq i \leq n
$$

where the sum runs over the connected components of the singular set $S$, $\operatorname{Res}_{\sigma_{i}}(\mathcal{F}, Z) \in H_{2 n-2 i}(S ; \mathbb{Z})$ is the corresponding Baum-Bott residue, and $\mu_{*}$ is given by the inclusion $H_{*}(S) \rightarrow H_{*}(M)$ followed by Alexander-Lefschetz duality $H_{*}(M) \simeq H^{*}(M, \partial M)$.

Proof. The residue formula is in Theorem 1.1 (in fact, for $\varphi=\sigma_{i}$, it is not necessary to take a basic connection), so we only need to prove the first statement in 1.4. The exact sequences $\left(1_{1}\right)$ and $\left(1_{3}\right)$ determine a resolution of $Q$;

$$
0 \rightarrow E_{q} \rightarrow \ldots \rightarrow E_{0} \rightarrow T M \rightarrow Q \rightarrow 0
$$

on $M$. Let $D$ be a connection for $\mathcal{F}$ on $M-S$ constructed as before and $\left(D_{q}^{\prime}, \ldots, D_{0}^{\prime}\right)$ connections for the $E_{i}$ 's on $M-S$ such that $\left(D_{q}^{\prime}, \ldots, D_{0}^{\prime}, D\right)$ are compatible with (13). Taking a basic connection $D_{-1}$ for $Q$ on $M-S$, let $\hat{D}^{\prime}$ be a connection for $T M$ on $M-S$ so that $\left(D, \hat{D}^{\prime}, D_{-1}\right)$ are compatible with $(1)$. Then $\left(D_{q}^{\prime}, \ldots, D_{0}^{\prime}, \hat{D}^{\prime}, D_{-1}\right)$ are compatible with the above sequence. We extend the connections $\hat{D}^{\prime}$ and $D_{i}^{\prime}$ to connections $\hat{D}$ and $D_{i}$ on $M$ so that the corresponding connections agree on $M-\Sigma$. Let $K_{i}, \hat{K}$ and $K_{-1}$ denote the curvatures of $D_{i}, \hat{D}$ and $D_{-1}$, respectively. The form $\sigma_{i}(\hat{K})$ defines the class $c_{i}(T M)$ and, in particular, if $n-k+1 \leq i \leq n$, it defines the class $c_{i}(T M, \mathbf{f})$. Also, if we define the $\rho_{i}$ 's by $\left(1_{4}\right)$ as before, the $2 i$-form 
$\rho_{i}$ defines the class $c_{i}(\mathcal{F})$ and, in particular, if $p-k+1 \leq i \leq n$, it defines the class $\tilde{c}_{i}(\mathcal{F})$. Finally, if we define the $\omega_{i}$ 's by

$$
1+\omega_{1}+\cdots+\omega_{n}=\left(1+\sigma_{1}(\hat{K})+\cdots+\sigma_{n}(\hat{K})\right) \cdot\left(1+\rho_{1}+\cdots+\rho_{n}\right)^{-1},
$$

the $2 i$-form $\omega_{i}$ defines the class $c_{i}(Q)$ and, in particular, if $n-p+1 \leq i \leq n$, it defines the class $\tilde{c}_{i}(Q)$. Hence we have the identity in 1.4 .

The Chern classes of $T M$ relative to the $k$-frame $\mathbf{f}$ were constructed above via differential geometry, but they can also be defined via obstruction theory as in $[\mathrm{Ke}]$. We do this in $\S 3$ below.

There are two interesting special cases: One is when $\mathcal{F}$ has isolated singularities and we have one vector field tangent to $\mathcal{F}$; This is discussed in $\S 4$ below. The other special case is when the number of vector fields $s_{1}, \ldots, s_{k}$ equals the dimension of $\mathcal{F}$, i.e., $k=p$, so that $s_{1}, \ldots, s_{k}$ determine a trivialization of $\mathcal{F}$ on a neighbourhood of the boundary. This happens, for instance, when the foliation is given by the action of $\mathbb{C}^{p}$. We prove that in this case the Chern classes of the normal sheaf are computable from the Chern classes of $M$ and $\mathcal{F}$, in the appropiate range:

1.5 Theorem. If $k=p$, for $i=1, \ldots, n$, define $\tilde{d}_{i} \in H^{2 i}(M, \partial M)$ by,

$$
\left(1+\tilde{d}_{1}+\cdots+\tilde{d}_{n}\right)\left(1+\tilde{c}_{1}(\mathcal{F})+\cdots+\tilde{c}_{n}(\mathcal{F})\right)=1
$$

Then

$$
\tilde{c}_{j}(Q)=\sum_{i=0}^{j-1} c_{i}(T M) \cdot \tilde{d}_{j-1}+\tilde{c}_{j}(T M)
$$

for $n-p+1 \leq j \leq n$.

Proof. If $k=p$, then all the $\rho_{i}$ 's have compact support. Observe that the form $1+\rho_{1}+\cdots+\rho_{n}$ is invertible, because it starts with 1 , so there exists $2 i$-forms $\tau_{i}$, $i=1, \ldots, n$, such that

$$
\left(1+\tau_{1}+\cdots+\tau_{n}\right)\left(1+\rho_{1}+\cdots+\rho_{n}\right)=1
$$

and the $\tau_{i}$ 's also have compact support. For each $i=1, \ldots, n$, define

$$
\tilde{d}_{i}=\left(\frac{\sqrt{-1}}{2 \pi}\right)^{i}\left[\tau_{i}\right]
$$

These are the classes stated in Theorem 1.5.

One also has in this case (when $k=p$ ) the following extension of 1.4, which is proved similarly, using $\left(1_{5}\right)$ and noting that all the $\rho_{i}$ 's have compact support as in 1.5 , so we only state the theorem. 
1.6 Theorem. Let $k=p$. For an arbitrary symmetric polynomial $\psi$ of degree $i>0$, there is a class $\tilde{\psi}(\mathcal{F})$ in $H^{2 i}(M, \partial M ; \mathbb{C})$ whose image by $j^{*}$ in $H^{2 i}(M ; \mathbb{C})$ is the class $\psi(\mathcal{F})$. Also, for a symmetric polynomial $\varphi$ of degree $d>n-p$, there are classes $\varphi(T M, \mathbf{f})$ and $\tilde{\varphi}(Q)$ in $H^{2 d}(M, \partial M ; \mathbb{C})$ whose images by $j^{*}$ in $H^{2 d}(M ; \mathbb{C})$ are the classes $\varphi(T M)$ and $\varphi(Q)$, respectively, and one has:

(i) $\tilde{\varphi}(Q)$ is the sum of residues localized at the singular set $S$ of $\mathcal{F}$,

$$
\tilde{\varphi}(Q)=\sum_{Z \subset S} \mu_{*} \operatorname{Res}_{\varphi}(Z, \mathcal{F})
$$

(ii) $\varphi(T M, \mathbf{f})$ is of the form

$$
\varphi(T M, \mathbf{f})=\sum_{i=1}^{d} \tilde{\psi}_{i}(\mathcal{F}) \cdot \varphi_{d-i}(Q)+\tilde{\varphi}(Q)
$$

where $\psi_{i}$ and $\varphi_{i}$ are symmetric polynomials of degree $i$, in particular $\psi_{d}=\varphi$ and $\varphi_{0}=1$.

1.7 Remarks. 1. Theorems 1.1, 1.4 and 1.6 generalize, respectively, Lemma 2.1 (a), Theorem I and Lemma 2.1 (b) in [SS].

2. We are assuming that the $k$-frame $\mathbf{f}=\left\{s_{1}, \ldots, s_{k}\right\}$ on $U$, a neighbourhood of $\partial M$, is tangent to $\mathcal{F}$; One may consider, more generally, frames $\mathbf{f}=\left\{s_{1}, \ldots, s_{q}, \nu_{1}, \ldots, \nu_{r}\right\}$ on $U$, where $\left\{s_{1}, \ldots, s_{q}\right\}$ is a $q$-frame tangent to $\mathcal{F}$ and $\left\{\nu_{1}, \ldots, \nu_{r}\right\}$ is an $r$-frame normal to $\mathcal{F} ;$ In this case $\left\{s_{1}, \ldots, s_{q}\right\}$ determine relative classes $c_{p}(\mathcal{F}, \mathbf{f}), \ldots, c_{p-q+1}(\mathcal{F}, \mathbf{f})$ of $\mathcal{F}$, while $\left\{\nu_{1}, \ldots, \nu_{r}\right\}$ determine relative classes $c_{n-p}(Q, \mathbf{f}), \ldots, c_{n-p-r+1}(Q, \mathbf{f})$. All these relative classes amount to determine relative classes $c_{n}(M, \mathbf{f}), \ldots, c_{n-k+1}(M, \mathbf{f})$, $k=q+r$, and one has similar formulae to those in Theorem 1.4 above. (Note that similar considerations are possible in $\S 2$ and $\S 3$ below.) If one tries to combine this with the residue theory for general characteristic polynomials, one needs to have on $Q$ a connection $D$ which is basic and trivial on the sub-bundle spanned by the normal vector fields $\nu_{1}, \ldots, \nu_{r}$. This means that all the $\nu_{i}$ 's must be holomorphic and the bracket $\left[u, \nu_{i}\right]$ must be in $\mathcal{F}$ for every $u \in \mathcal{F}$, i.e., the $\nu_{i}$ 's define infinitesimal automorphisms of $\mathcal{F}$. Such vector fields are $\Gamma$-vector fields for $\mathcal{F}$ in the sense of [He].

\section{$\S 2$ The Euler class for singular foliations on $C^{\infty}$ manifolds}

We refer to [St,MS,Ke] for background on characteristic classes. Let $M$ be a compact, oriented, $C^{\infty}$ manifold of dimension $n>0$ and $E$ a vector bundle of rank $q$ over $M, 0<q \leq n$. The Euler class of $E, e(E) \in H^{q}(M ; \mathbb{Z})$, is the first. possibly non-zero obstruction for constructing a cross section of $E$. For instance, if $M$ has empty boundary and $E=T M$, then $e(E)[M]=\chi(M)$. If the bundle $E$ is the direct sum of $E_{1}$ and $E_{2}$, then one has the Whitney formula:

$$
e(E)=e\left(E_{1}\right) \cdot e\left(E_{2}\right)
$$


We are also interested in considering extensions to $M$ of cross sections given on a sub-complex of $M$. More precisely, assume $M$ has non-empty boundary $\partial M$, and let $s$ be a cross section of $\left.E\right|_{\partial M}$, the restriction of $E$ to $\partial M$. The first possibly non-zero obstruction for extending $s$ to the interior of $M$ is a class $e(E, s)$ in $H^{q}(M, \partial M ; \mathbb{Z})$, called the Euler class of $E$ relative to $s$. The image of $e(E, s)$ in the absolute cohomology is $e(E)$ independently of $s$, but as a relative class $e(E, s)$ does depend on the choice of $s$ on $\partial M$, generally speaking. For instance, if $E$ is $T M$ and $s$ is a cross section of $\left.T M\right|_{\partial M}$, then one has,

$$
e(T M, s)[M]=\operatorname{Ind}(s, M),
$$

where $\operatorname{Ind}(s, M)$ is the total Poincaré-Hopf index of $s$ in $M$.

Let $\mathcal{D}$ be a $C^{\infty}$ field of oriented $p$-planes on $M$ with singular set $S$ contained in the interior of $M$. By this we mean a smooth $p$-dimensional sub-bundle of $T M$ on $M-S$, where $S$ is a subcomplex for some triangulation of $M$. One has the exact sequence of the pair $(M, M-S)$,

$$
\cdots \rightarrow H^{p}(M, M-S) \rightarrow H^{p}(M) \stackrel{i^{*}}{\rightarrow} H^{p}(M-S) \rightarrow H^{p+1}(M, M-S) \rightarrow \cdots .
$$

By Alexander duality one has,

$$
H^{p}(M, M-S) \simeq H_{n-p}(S) .
$$

Thus, if $H_{n-p}(S) \simeq H_{n-p-1}(S) \simeq 0$, then $i^{*}$ is an isomorphism. Hence, there exists a unique class in $H^{p}(M)$ whose image in $H^{p}(M-S)$ is the Euler class $e(\mathcal{D})$. Similarly, if we denote by $\mathcal{D}^{\perp}$ the orthogonal complement of $\mathcal{D}$ on $M-S$, with respect to some riemannian metric, then the Euler class of $\mathcal{D}^{\perp}$ on $M-S$ lives in $H^{n-p}(M-S ; \mathbb{Z})$, which is isomorphic to $H^{n-p}(M ; \mathbb{Z})$ whenever $H_{p}(S) \simeq H_{p-1}(S) \simeq$ 0 .

The following definition is given in $[\mathrm{T}]$ for fields of 2-planes with isolated singularities, but the definition is appropriate in general.

2.1 Definition. Let $\mathcal{D}$ be a field of $p$-planes on $M-S$. If $H_{n-p}(S) \simeq H_{n-p-1}(S) \simeq$ 0 , then the Euler class of $\mathcal{D}$ is the unique cohomology class $e(\mathcal{D}) \in H^{p}(M ; \mathbb{Z})$ whose image in $H^{p}(M-S ; \mathbb{Z})$ is the usual Euler class of $\mathcal{D}$ on $M-S$. If $H_{p}(S) \simeq$ $H_{p-1}(S) \simeq 0$, then the Euler class of $\mathcal{D}^{\perp}$ is the unique class in $H^{n-p}(M ; \mathbb{Z})$ whose image in $H^{p}(M-S ; \mathbb{Z})$ is the usual Euler class of $\mathcal{D}^{\perp}$ on $M-S$.

For instance, if the dimension of each connected component of $S$ is strictly less than $n-p-1$, then the Euler class of $\mathcal{D}$ is well defined on all of $M$. If the dimension of each component of $S$ is strictly less than $p-1$, then the Euler class of $\mathcal{D}^{\perp}$ is defined on all of $M$.

Let $Z$ be a connected component of $S$, and let $N$ be a neighbourhood of $Z$ with smooth boundary $K$. Suppose we have on $N-Z$ two non-singular vector fields $v$ and $s$, both contained in $\mathcal{D}$. One has the following lemma: 
2.2 Lemma. If either $H_{n-p}(K ; \mathbb{Z})$ or $H_{p-1}(K ; \mathbb{Z})$ vanishes, then $v$ and $s$ have the same total index in $N$.

Proof. We can assume that $N$ is a regular neighbourhood of $Z$ with smooth boundary $K$. We take another regular neighbourhood contained in the interior of $N$ with smooth boundary $L$. Then $K$ and $L$ bound a manifold $C$ diffeomorphic to the cylinder $K \times I$. We define a vector field $X$ on $\partial C$ as being $v$ on $K$ and $s$ on $L$. Then $X$ can be extended to the interior of $C$ with no singularities if and only if its total index in $C$ is 0 . Let $\mathcal{D}^{\perp}$ be as above, and let $e\left(\mathcal{D}^{\perp}\right)$ be the Euler class of $\mathcal{D}^{\perp}$ on $C$, let $e(C, X)$ be the Euler class of $C$ relative to $X$, and let $e(\mathcal{D}, X)$ be the relative Euler class of $\mathcal{D}$ on $C$. One has

$$
\operatorname{Ind}(X, C)=e(C, X)[C]=e(\mathcal{D}, X) \cdot e\left(\mathcal{D}^{\perp}\right)[C] .
$$

One has $e(\mathcal{D}, X) \in H^{p}(C, \partial C ; \mathbb{Z}) \simeq H_{n-p}(K ; \mathbb{Z})$ and $e\left(\mathcal{D}^{\perp}\right) \in H^{n-p}(C ; \mathbb{Z}) \simeq$ $H_{p-1}(K ; \mathbb{Z})$. Hence, if either $H_{n-p}(K ; \mathbb{Z})$ or $H_{p-1}(K ; \mathbb{Z})$ vanishes, then $\operatorname{Ind}(X, C)=$ 0 and 2.2 follows.

2.3 Definition. Let $\mathcal{D}$ be as above and let $Z$ be a connected component of the singular set $S$. Let $N$ be a regular neighbouhood of $Z$ with smooth boundary $K$. Suppose that either $H_{n-p}(K ; \mathbb{Z}) \simeq 0$ or $H_{p-1}(K ; \mathbb{Z}) \simeq 0$, and suppose also that there exists a non-singular vector field $v$ on $K$ which is contained in $\mathcal{D}$. Then the topological Euler residue of $\mathcal{D}$ at $Z, \operatorname{TRes}_{e}(\mathcal{D}, Z) \in \mathbb{Z}$, is the total index of $v$ on $N$.

Lemma 2.2 implies that $\operatorname{TRes}_{e}(\mathcal{D}, Z)$, when it is defined, depends only on $\mathcal{D}$ and not on the choice of the vector field $v$. Note that from the exact sequence

$$
\cdots \rightarrow H^{p-1}(Z) \rightarrow H_{n-p}(K) \rightarrow H_{n-p}(Z) \rightarrow \cdots,
$$

we see that, if, for example, the dimension of $Z$ is less than $n-p$ and $p-1$, we have $H_{n-p}(K) \simeq 0$. Considering a similar sequence, we have also $H_{p-1}(K) \simeq 0$ under the same condition.

2.4 Theorem. Let $M$ be a closed, oriented, $C^{\infty} n$-manifold and let $\mathcal{D}$ be a $C^{\infty}$ field of oriented p-planes on $M$, singular on a set $S$ which is a simplicial sub-complex of $M$ for some triangulation. Let $Z_{1}, \ldots, Z_{r}$ be the connected components of $S$. Assume that for all $\alpha=1, \ldots, r$ one has:

(i) The dimension $\ell_{\alpha}$ of $Z_{\alpha}$ satisfies $\ell_{\alpha}<n-p-1$ and $\ell_{\alpha}<p-1$, and

(ii) There exists a neighbourhood $N_{\alpha}$ of $Z_{\alpha}$, with a non-singular vector field on $N_{\alpha}-Z_{\alpha}$ contained in $\mathcal{D}$. Then :

(a) The Euler classes of both $\mathcal{D}$ and $\mathcal{D}^{\perp}$ are well defined on $M$.

(b) The topological Euler residue of $\mathcal{D}$ is well defined at each $Z_{\alpha}$.

(c) The Euler-Poincaré characteristic of $M$ is given by:

$$
\chi(M)=e(\mathcal{D}) \cdot e\left(\mathcal{D}^{\perp}\right)[M]+\sum_{\alpha=1}^{r} \operatorname{TRes}_{e}\left(\mathcal{D}, Z_{\alpha}\right)
$$


where $[M]$ is the orientation cycle of $M$.

Proof. Statements (a) and (b) are already proved. For (c), we observe that one has:

$$
\chi(M)=e(M)[M]=e(M-\operatorname{Int} N, v)[M-\operatorname{Int} N]+e(N, v)[N]
$$

where $N=\cup N_{\alpha}$. On $M-\operatorname{Int} N, \mathcal{D}$ and $\mathcal{D}^{\perp}$ are vector bundles, hence one has,

$$
\begin{aligned}
e(M-\operatorname{Int} N, v)[M-\operatorname{Int} N] & =e\left(\left.\mathcal{D}\right|_{M-\operatorname{Int} N}, v\right) \cdot e\left(\left.\mathcal{D}^{\perp}\right|_{M-\operatorname{Int} N}\right)[M-\operatorname{Int} N] \\
& =e(\mathcal{D}) \cdot e\left(\mathcal{D}^{\perp}\right)[M]
\end{aligned}
$$

because the Euler classes $e(\mathcal{D})$ and $e\left(\mathcal{D}^{\perp}\right)$ have support on $M-\operatorname{Int} N$. By definition one has,

$$
e(N, v)[N]=\sum_{i=1}^{r} \operatorname{TRes}_{e}\left(\mathcal{D}, Z_{\alpha}\right)
$$

Statement (c) of 2.4 follows from equations $\left(2_{1}\right),\left(2_{2}\right)$ and $\left(2_{3}\right)$.

The extension of Theorem 2.4 to manifolds with boundary is immediate, with essentially the same proof.

2.5 Theorem. Let $M$ be a compact, oriented, $C^{\infty} n$-manifold with boundary $\partial M$. Let $\mathcal{D}$ be a $C^{\infty}$ field of oriented $p$-planes on $M$, singular on a set $S$ contained in the interior of $M$, which is a simplicial sub-complex of $M$ for some triangulation. Let $Z_{1}, \ldots, Z_{r}$ be the connected components of $S$. Assume that for all $\alpha=1, \ldots, r$ one has:

(i) The dimension $\ell_{\alpha}$ of $Z_{\alpha}$ satisfies $\ell_{\alpha}<n-p-1$ and $\ell_{\alpha}<p-1$, and

(ii) There exists a neighbourhood $N_{\alpha}$ of $Z_{\alpha}$, with a non-singular vector field on $N_{\alpha}-Z_{\alpha}$ contained in $\mathcal{D}$. Then :

(a) The Euler classes of both $\mathcal{D}$ and $\mathcal{D}^{\perp}$ are defined on all of $M$.

(b) The Topological Euler Residue of $\mathcal{D}$ is well defined at each $Z_{\alpha}$.

(c) If $X$ is a non-singular vector field on a neighbouhood of $\partial M$ and contained in $\mathcal{D}$, then the total index of $X$ in $M$ is given by:

$$
\operatorname{Ind}(X, M)=e(\mathcal{D}, X) \cdot e\left(\mathcal{D}^{\perp}\right)[M]+\sum_{\alpha=1}^{r} \operatorname{TRes}_{e}\left(\mathcal{D}, Z_{\alpha}\right)
$$

where $e(\mathcal{D}, X) \in H^{p}(M, \partial M ; \mathbb{Z})$ is the unique cohomology class that maps to the Euler class of $\mathcal{D}$ on $M-S$ relative to the vector field $X$ on $\partial M$. 


\section{§3 Characteristic classes for singular foliations on $C^{\infty}$ manifolds}

We now discuss the Chern classes for fields of complex planes with singularities. We remark that all the results in this section are easily adapted to fields of real planes as in $\$ 2$ above, replacing the Chern classes by the Stiefel-Whitney classes. The relationship with the residues in [SW] will be discussed elsewhere.

Let $E$ be a complex vector bundle of rank $q$ over $M$, a smooth manifold of dimension $m, 2 q \leq m$. The top Chern class of $E, c_{q}(E) \in H^{2 q}(M ; \mathbb{Z})$, is the Euler class of $E$. To construct the Chern class $c_{q-1}(E) \in H^{2 q-2}(M ; \mathbb{Z})$ we let $\mathcal{W}_{2, q}$ be the fibre bundle over $M$ whose fibre at each point $x \in M$ is the Stiefel manifold $W_{2, q}$ of complex 2-frames in the fibre $E_{x}$ of $E$ over $x$; The manifold $W_{2, q}$ is $(2 q-4)$-connected, and $\pi_{2 q-3}\left(W_{2, q}\right) \simeq \mathbb{Z}$, by Bott's computations of the homotopy groups of the classical groups (see $[\mathrm{Hu}]$ ). Hence, the first possibly nonzero obstruction for constructing a section of $\mathcal{W}_{2, q}$ lives in $H^{2 q-2}(M ; \mathbb{Z})$, and this is $c_{q-1}(E)$ by definition. In general, to construct the class $c_{q-i}(E) \in H^{2 q-2 i}(M ; \mathbb{Z})$, $i=0, . ., q-1$, we form the fibre bundle $\mathcal{W}_{i+1, q}$ whose fibre at each point is the Stifel Manifold of complex $(i+1)$-frames in the fibre $E_{x} \simeq \mathbb{C}^{q} ; c_{q-i}$ is the first possibly non-zero obstruction for constructing a section of $\mathcal{W}_{i+1, q}$. If the bundle $E$ is the direct sum of two complex bundles $E_{1}$ and $E_{2}$, then one has the Whitney relations: For each $k=1, \ldots, q$ one has,

$$
c_{k}(E)=\sum_{i+j=k} c_{i}\left(E_{1}\right) \cdot c_{j}\left(E_{2}\right) .
$$

If $M$ has boundary $\partial M$ and one has a complex $k$-frame $\mathbf{f}$ over the boundary, then one has representatives of the Chern classes of $E$ in the relative cohomology, $c_{i}(E, \mathbf{f}) \in H^{2 i}(M, \partial M ; \mathbb{Z})$, whose image in $H^{*}(M ; \mathbb{Z})$ are the usual Chern classes. These are the Chern classes of $E$ relative to the $k$-frame $\mathbf{f}$.

Now suppose $M$ is a compact, almost complex manifold of real dimension $2 n$, and $\mathcal{D}$ is a smooth field of complex $p$-planes on $M$, singular at a set $S$ as in $\S 2$ above. Let $\mathcal{D}^{\perp}$ be the complex orthogonal complement of $\mathcal{D}$ on $M-S$, with respect to some hermitian metric. Thus $\mathcal{D}^{\perp}$ is a field of complex $(n-p)$-planes on $M$, singular at $S$. On $M-S$ one has the usual Chern classes of $\mathcal{D}$ and $\mathcal{D}^{\perp}$, $c_{i}\left(\left.\mathcal{D}\right|_{M-S}\right) \in H^{2 i}(M-S)$ for $i=1, \ldots, p$, and $c_{j}\left(\left.\mathcal{D}^{\perp}\right|_{M-S}\right) \in H^{2 j}(M-S)$ for $j=1, \ldots, n-p$. As before, one has the exact sequence,

$$
\cdots \rightarrow H^{2 i}(M, M-S) \rightarrow H^{2 i}(M) \rightarrow H^{2 i}(M-S) \rightarrow H^{2 i+1}(M, M-S) \rightarrow \cdots
$$

Hence, if $H^{2 i}(M, M-S) \simeq H^{2 i+1}(M, M-S) \simeq 0$, then in the appropiate ranges of dimensions, any class $c_{i}\left(\left.\mathcal{D}\right|_{M-S}\right)$ or $c_{i}\left(\left.\mathcal{D}^{\perp}\right|_{M-S}\right)$ will extend uniquely to a cohomology class on all of $M$. We observe that Alexander duality implies:

$$
H^{2 i}(M, M-S) \simeq H_{2 n-2 i}(S), \quad H^{2 i+1}(M, M-S) \simeq H_{2 n-2 i-1}(S) .
$$

Therefore one has the following lemma: 
3.1 Lemma. Let $Z_{1}, \ldots, Z_{r}$ be the connnected components of the singular set $S$, and let $\ell_{\alpha}=\operatorname{dim} Z_{\alpha}$ for $\alpha=1, \ldots, r$. If

$$
\ell_{\alpha}<2 n-2 p-1
$$

for every $\alpha=1, \ldots, r$, then all the Chern classes of $\mathcal{D}$ on $M-S$ extend uniquely to cohomology classes $c_{j}(\mathcal{D}) \in H^{2 j}(M ; \mathbb{Z}), j=1, \ldots, p$. If

$$
\ell_{\alpha}<2 p-1
$$

for every $\alpha=1, \ldots, r$, then all the Chern classes of $\mathcal{D}^{\perp}$ on $M-S$ extend uniquely to cohomology classes $c_{j}\left(\mathcal{D}^{\perp}\right) \in H^{2 j}(M ; \mathbb{Z}), j=1, \ldots, n-p$.

Assume now that we have a complex 2-frame $f=\left\{s_{1}, s_{2}\right\}$, on $N-Z$, where $Z$ is a component of $S$ and $N$ is a regular neighbourhood of $Z$ with smooth boundary $K$. This 2 -frame defines relative Chern classes $c_{j}(N, \mathbf{f}) \in H^{2 j}(N, K ; \mathbb{Z}), j=n, n-1$.

3.2 Proposition. Let $\left\{s_{1}, s_{2}\right\}$ be as above and let $\mathbf{g}=\left\{v_{1}, v_{2}\right\}$ be another complex 2 -frame on $N-Z$. Assume that both frames are contained in $\mathcal{D}$. Let $\ell$ be the dimension of $Z$. If $\ell<2 n-2 p$ and $\ell<2 p-1$, then the relative Chern classes defined by $\mathbf{f}$ and $\mathbf{g}$ coincide:

$$
c_{j}(N, \mathbf{f})=c_{j}(N, \mathbf{g})
$$

for $j=n, n-1$.

Proof. For $j=n, 3.2$ was proved in $\S 2$ above; We now prove 3.2 for the Chern class $c_{n-1}$. Let $N^{\prime}$ be a regular neighbourhood of $Z$ contained in the interior of $N$ and with smooth boundary $L$. Then $K$ and $L$ bound a cylinder $C$ diffeomorphic to $K \times I$, and we have a 2 -frame $\Phi$ on $\partial C$, given by $\mathbf{f}$ on $K$ and $\mathbf{g}$ on $L$. One has,

$$
c_{n-1}(C, \Phi)=c_{p-1}(\mathcal{D}, \Phi) \cdot c_{n-p}\left(\mathcal{D}^{\perp}\right)+c_{p}(\mathcal{D}, \Phi) \cdot c_{n-p-1}\left(\mathcal{D}^{\perp}\right)
$$

The class $c_{n-p}\left(\mathcal{D}^{\perp}\right)$ lives in $H^{2 n-2 p}(C) \simeq H^{2 n-2 p}(K) \simeq H_{2 p-1}(K)$, which vanishes because $\operatorname{dim} Z<2 n-2 p, 2 p-1$ (see the remark right before Theorem 2.4). Hence, $c_{p-1}(\mathcal{D}, \Phi) \cdot c_{n-p}\left(\mathcal{D}^{\perp}\right)=0$. Similarly, $c_{p}(\mathcal{D}, \Phi) \in H^{2 p}(C, \partial C) \simeq H^{2 p-1}(K)=0$. Hence,

$$
c_{n-1}(C, \Phi)=0
$$

and 3.2 follows.

Just as in $\S 2$ above, lemma 3.2 allows us to define topological residues corresponding to the Chern classes $c_{n}$ and $c_{n-1}$ : 
3.3 Definition. Suppose $Z$ is a component of $S$ such that:

(i) There exists a 2-frame $\mathbf{f}$ on the boundary $K$ of a regular neighbouhood $N$ of $Z$ contained in $\mathcal{D}$, and

(ii) $H_{2 n-2 p}(K ; \mathbb{Z}) \simeq H_{2 p-1}(K ; \mathbb{Z}) \simeq 0$. Then one has well defined topological residues of $\mathcal{D}$ at $Z$ corresponding to the Chern classes $c_{n}$ and $c_{n-1}$,

$$
\begin{aligned}
& \operatorname{TRes}_{c_{n}}(\mathcal{D}, Z) \in H_{0}(Z ; \mathbb{Z}) \simeq \mathbb{Z}, \\
& \operatorname{TRes}_{c_{n-1}}(\mathcal{D}, Z) \in H_{2}(Z ; \mathbb{Z}),
\end{aligned}
$$

respectively. These are, by definition, the classes in

$$
H_{2 i}(Z) \simeq H_{2 i}(N) \simeq H^{2 n-2 i}(N, K), \quad i=0,1,
$$

determined by the Chern classes of $N$ relative to the 2-frame $\mathbf{f}$.

Assume now that we have a complex $k$-frame $\mathbf{f}=\left\{s_{1}, \ldots, s_{k}\right\}$ on $N-Z$, where $Z$ is a component of $S$ and $N$ is a regular neighbourhood of $Z$ with smooth boundary $K$. This $k$-frame defines relative Chern classes $c_{j}(N, \mathbf{f}) \in H^{2 j}(N, K ; \mathbb{Z})$, $j=n, \ldots, n-k+1$. If $\mathbf{f}$ is contained in $\mathcal{D}$, and if $\mathbf{g}$ is another such $k$-frame, then the difference between the Chern classes relative to $\mathbf{f}$ and $\mathbf{g}$ is given by the Chern classes of $C$ relative to the boundary $\partial C$, where $C$ is a cylinder as above and we have on its boundary $K \cup L$, a $k$-frame $\Phi$ which is $\mathbf{f}$ on $K$ and $\mathbf{g}$ on $L$. Concerning the classes $c_{n-2}, \ldots, c_{n-k+1}$, one has:

$$
\begin{aligned}
c_{n-2}(C, \Phi)= & c_{p}(\mathcal{D}, \Phi) \cdot c_{n-p-2}\left(\mathcal{D}^{\perp}\right)+c_{p-1}(\mathcal{D}, \Phi) \cdot c_{n-p-1}\left(\mathcal{D}^{\perp}\right) \\
& +c_{p-2}(\mathcal{D}, \Phi) \cdot c_{n-p}\left(\mathcal{D}^{\perp}\right) \\
c_{n-3}(C, \Phi)= & c_{p}(\mathcal{D}, \Phi) \cdot c_{n-p-3}\left(\mathcal{D}^{\perp}\right)+c_{p-1}(\mathcal{D}, \Phi) \cdot c_{n-p-2}\left(\mathcal{D}^{\perp}\right) \\
& +c_{p-2}(\mathcal{D}, \Phi) \cdot c_{n-p-1}\left(\mathcal{D}^{\perp}\right)+c_{p-3}(\mathcal{D}, \Phi) \cdot c_{n-p}\left(\mathcal{D}^{\perp}\right)
\end{aligned}
$$

and so on, up to

$$
c_{n-k+1}(C, \Phi)=\sum_{i=0}^{k-1} c_{p-i}(\mathcal{D}, \Phi) \cdot c_{n-k-p+i+1}\left(\mathcal{D}^{\perp}\right) .
$$

Let $\ell$ be the maximal dimension of the components of $S$. If $\ell<2 p-1,2 n-2 p-1$, then in the above formulae, the first and last terms on the right hand side vanish, but the middle terms may not be zero. Thus we need to ask for more: If $\ell<2 p-3$, $2 n-2 p-2$, then $c_{n-2}(N, \mathbf{f})$ and $c_{n-3}(N, \mathbf{f})$ are also independent of $\mathbf{f}$, they depend only on $\mathcal{D}$ near $Z$, and one has well defined topological residues of $\mathcal{D}$ at $Z$,

$$
\begin{aligned}
& \operatorname{TRes}_{c_{n-2}}(\mathcal{D}, Z) \in H_{4}(Z ; \mathbb{Z}) \simeq H^{2 n-4}(N, K ; \mathbb{Z}), \\
& \operatorname{TRes}_{c_{n-3}}(\mathcal{D}, Z) \in H_{6}(Z ; \mathbb{Z}) \simeq H^{2 n-6}(N, K ; \mathbb{Z}),
\end{aligned}
$$


and so on.

We summarize the previous discussion in the following theorem. We let $M$ be a closed, $2 n$-dimensional almost-complex manifold, and let $\mathcal{D}$ be a field of complex $p$-planes on $M$, whose singular locus $S$ is a simplicial sub-complex of $M$ for some triangulation. Let $Z_{1}, \ldots, Z_{r}$ be the connected components of $S$, and let $\ell$ be the maximal of the dimensions of the $Z_{\alpha}$ 's.

3.4 Theorem. If $\ell<2 p-1,2 n-2 p-1$ then:

(i) One has Chern classes of $\mathcal{D}$ and $\mathcal{D}^{\perp}$,

$$
c_{j}(\mathcal{D}) \in H^{2 j}(M ; \mathbb{Z}), j=1, \ldots, p, \text { and } c_{i}\left(\mathcal{D}^{\perp}\right) \in H^{2 i}(M ; \mathbb{Z}), i=1, \ldots, n-p,
$$

uniquely characterized by the fact that they map to the usual Chern classes of these bundles under the inclusion homomorphism $H^{*}(M) \rightarrow H^{*}(M-S)$.

(ii) If there exists a vector field $v_{\alpha}$ on a neighbourhood $N_{\alpha}$ of each $Z_{\alpha}$, non-singular on $N_{\alpha}-Z_{\alpha}$ and contained in $\mathcal{D}$, then there exists for each $Z_{\alpha}$ a well defined homology class $\operatorname{TRes}_{c_{n}}\left(\mathcal{D}, Z_{\alpha}\right) \in H_{0}\left(Z_{\alpha} ; \mathbb{Z}\right) \simeq \mathbb{Z}$, which depends only on $\mathcal{D}$, and such that:

$$
c_{n}(M)[M]=c_{p}(\mathcal{D}) \cdot c_{n-p}\left(\mathcal{D}^{\perp}\right)[M]+\sum_{\alpha=1}^{r} \operatorname{TRes}_{c_{n}}\left(\mathcal{D}, Z_{\alpha}\right) .
$$

(iii) If, moreover, there exists on each $N_{\alpha}-Z_{\alpha}$ a 2-frame contained in $\mathcal{D}$, then there exists a well defined homology class $\operatorname{TRes}_{c_{n-1}}\left(\mathcal{D}, Z_{\alpha}\right) \in H_{2}\left(Z_{\alpha} ; \mathbb{Z}\right)$, which depends only on $\mathcal{D}$, and if $\mu_{*}$ is the composition $H_{2}\left(Z_{\alpha} ; \mathbb{Z}\right) \rightarrow H_{2}(M ; \mathbb{Z}) \rightarrow H^{2 n-2}(M ; \mathbb{Z})$, then

$$
c_{n-1}(M)=c_{p}(\mathcal{D}) \cdot c_{n-p-1}\left(\mathcal{D}^{\perp}\right)+c_{p-1}(\mathcal{D}) \cdot c_{n-p}\left(\mathcal{D}^{\perp}\right)+\sum_{\alpha=1}^{r} \mu_{*} \operatorname{TRes}_{c_{n-1}}\left(\mathcal{D}, Z_{\alpha}\right)
$$

(iv) If the dimension of each $Z_{\alpha}$ is even smaller, with respect to $p$ and $n-p$, and if there are more linearly independent vector fields around each $Z_{\alpha}$ contained in $\mathcal{D}$, one has topological residues for lower Chern classes, and the corresponding formulae relating these residues with the Chern classes of $M$.

We are also interested in the case when $M$ is a compact, almost-complex manifold of dimension $2 n$, with non-empty boundary $\partial M$. Let $\mathcal{D}$ be a field of complex $p$-planes on $M$, whose singular locus $S$ is a simplicial sub-complex of the interior of $M$. If the dimension of each component $Z_{1}, \ldots, Z_{r}$ of $S$ is smaller than $2 p-1$ and $2 n-2 p-1$, then one has Chern classes of $\mathcal{D}$ and $\mathcal{D}^{\perp}$ as above,

$$
c_{j}(\mathcal{D}) \in H^{2 j}(M ; \mathbb{Z}), j=1, \ldots, p, \text { and } c_{i}\left(\mathcal{D}^{\perp}\right) \in H^{2 i}(M ; \mathbb{Z}), i=1, \ldots, n-p,
$$

characterized by the fact that they are the usual Chern classes on $M-S$. If one has a $k$-frame $X=\left(X_{1}, \ldots, X_{k}\right)$ on a neighbourhood $U$ of $\partial M$ and contained in $\mathcal{D}$, then one has the corresponding relative Chern classes $c_{n-i}(M, X)$ and $c_{p-i}(\mathcal{D}, X)$, $i=0, \ldots, k-1$. 
3.5 Theorem. With the above hypotheses and notation:

(i) If $X$ is a 1-frame and if there exists a vector field $v_{\alpha}$ on a neighbourhood $N_{\alpha}$ of each $Z_{\alpha}$, non-singular on $N_{\alpha}-Z_{\alpha}$ and contained in $\mathcal{D}$, then there exists for each $Z_{\alpha}$, a well defined homology class $\operatorname{TRes}_{c_{n}}\left(\mathcal{D}, Z_{\alpha}\right) \in H_{0}\left(Z_{\alpha} ; \mathbb{Z}\right) \simeq \mathbb{Z}$, which depends only on $\mathcal{D}$, and such that:

$$
c_{n}(M, X)[M]=c_{p}(\mathcal{D}, X) \cdot c_{n-p}\left(\mathcal{D}^{\perp}\right)[M]+\sum_{\alpha=1}^{r} \operatorname{TRes}_{c_{n}}\left(\mathcal{D}, Z_{\alpha}\right)
$$

(ii) If, moreover, $X$ is a 2-frame, and if there exists on each $N_{\alpha}-Z_{\alpha}$ a 2-frame contained in $\mathcal{D}$, then there exist well defined homology classes $\operatorname{TRes}_{c_{n-1}}\left(\mathcal{D}, Z_{\alpha}\right) \in$ $H_{2}\left(Z_{\alpha} ; \mathbb{Z}\right)$, which depends only on $\mathcal{D}$, and if $\mu_{*}$ is the composition $H_{2}\left(Z_{\alpha} ; \mathbb{Z}\right) \rightarrow$ $H_{2}(M ; \mathbb{Z}) \rightarrow H^{2 n-2}(M ; \mathbb{Z})$, then

$$
\begin{aligned}
c_{n-1}(M, X)= & c_{p}(\mathcal{D}, X) \cdot c_{n-p-1}\left(\mathcal{D}^{\perp}\right)+c_{p-1}(\mathcal{D}, X) \cdot c_{n-p}\left(\mathcal{D}^{\perp}\right) \\
& +\sum_{\alpha=1}^{r} \mu_{*} \operatorname{TRes}_{c_{n-1}}\left(\mathcal{D}, Z_{\alpha}\right)
\end{aligned}
$$

(iii) If $X$ is a $k$-frame, then one has a statement similar to part (iv) of Theorem 3.4 above.

\section{\$4 Applications to singular holomorphic foliations}

We now let $M$ be a complex $n$-manifold, and $\mathcal{F}$ is a $p$-dimensional holomorphic foliation with singular set $S$. Let $Z_{1}, \ldots, Z_{r}$ be the connected components of $S$. Assume first that $M$ is compact. Let $c_{j}(\mathcal{F}), c_{j}(Q) \in H^{2 j}(M ; \mathbb{C}), j=1, \ldots, n$, be respectively, the Chern classes of $\mathcal{F}$ and $Q$, the normal sheaf. The following theorem is immediate from Theorem 3.4 above. We note that in this case, each $Z_{\alpha}$ is a complex analytic space; Let $\ell_{\alpha}$ be the complex dimension of $Z_{\alpha}$.

4.1 Theorem. (i) If $\ell_{\alpha}<n-p$ and $\ell_{\alpha}<p$ for all $\alpha=1, \ldots, r$, then the Chern classes

$$
c_{1}(\mathcal{F}), . ., c_{p}(\mathcal{F}) \text { and } c_{1}(Q), \ldots, c_{n-p}(Q),
$$

are characterized by the fact that on $M-S$, they are the usual Chern classes of $T \mathcal{F}$ and $N \mathcal{F}$, the bundles tangent and normal to $\mathcal{F}$, respectively. In particular, these classes are all integral.

(ii) If for each component $Z_{\alpha}$ of $S$ there exist a neighbourhood $N_{\alpha}$ and and a non-singular $C^{\infty}$ vector field $v_{\alpha}$ on $N_{\alpha}-Z_{\alpha}$ tangent to $\mathcal{F}$, then:

$$
\chi(M)=c_{n}(M)[M]=c_{p}(\mathcal{F}) \cdot c_{n-p}(Q)[M]+\sum_{\alpha=1}^{r} \operatorname{TRes}_{c_{n}}\left(\mathcal{F}, Z_{\alpha}\right)
$$


and one has:

$$
\sum_{\alpha=1}^{r} \operatorname{TRes}_{c_{n}}\left(\mathcal{F}, Z_{\alpha}\right)=\left(\sum_{i=0}^{p-1} c_{i}(\mathcal{F}) \cdot c_{n-i}(Q)+\sum_{i=p+1}^{n} c_{i}(\mathcal{F}) \cdot c_{n-i}(Q)\right)[M] .
$$

(iii) If on each $N_{\alpha}-Z_{\alpha}$ one has a 2 -frame tangent to $\mathcal{F}$, then

$$
c_{n-1}(M)=c_{p}(\mathcal{F}) \cdot c_{n-p-1}(Q)+c_{p-1}(\mathcal{F}) \cdot c_{n-p}(Q)+\sum_{\alpha=1}^{r} \mu_{*} \operatorname{TRes}_{c_{n-1}}\left(\mathcal{F}, Z_{\alpha}\right) .
$$

(iv) If on each $N_{\alpha}-Z_{\alpha}$ one has a $k$-frame tangent to $\mathcal{F}, p>k>2$, and if the dimension of all $Z_{\alpha}$ 's is even smaller with respect to $p$ and $n-p$, then one has similar formulae for the lower Chern classes:

$$
c_{k}(M)=\sum_{i=0}^{k} c_{i}(\mathcal{F}) \cdot c_{k-i}(Q)+\sum_{\alpha=1}^{r} \mu_{*} \operatorname{TRes}_{c_{k}}\left(\mathcal{F}, Z_{\alpha}\right)
$$

One also has the equivalent of 4.1 for manifolds with boundary, which follows from 3.5. Let $M$ be an open complex $n$-manifold which is relatively compact in a complex manifold; let $\partial M$ be the boundary of $M$. Assume we have a non-singular vector field $X$ on a neighbourhood $U \subset M$ of $\partial M$, which is tangent to $\mathcal{F}$, a $p$ dimensional holomorphic foliation on $M$ whose singular locus $S$ does not intersect $U$.

4.2 Theorem. (i) If the complex dimension of $S$ is less than $p$ and $n-p$, then the relative Chern class $c_{p}(\mathcal{F}, X)$, and the Chern classes $c_{i}(\mathcal{F}), i=1, \ldots, p-1$ and $c_{i}(Q), i=1, \ldots, n-p$, are characterized by being the usual Chern classes of the bundles $T \mathcal{F}$ and $N \mathcal{F}$ on $M-S$. Hence they are integral.

(ii) If there exists a neighbourhood $N$ of $S$ and a continuous, non-singular vector field $v$ on $N-S$ tangent to $\mathcal{F}$, then

$$
\operatorname{Ind}(X, M)=c_{n}(M, X)[M]=c_{p}(\mathcal{F}, X) \cdot c_{n-p}(Q)[M]+\sum_{\alpha=1}^{r} \operatorname{TRes}_{c_{n}}\left(\mathcal{F}, Z_{\alpha}\right)
$$

and

$$
\sum_{\alpha=1}^{r} \operatorname{TRes}_{c_{n}}\left(\mathcal{F}, Z_{\alpha}\right)=\left(\sum_{i=0}^{p-1} c_{i}(\mathcal{F}) \cdot \tilde{c}_{n-i}(Q)+\sum_{i=p+1}^{n} \tilde{c}_{i}(\mathcal{F}) \cdot c_{n-i}(Q)\right)[M],
$$

is the contribution of the singular set.

(iii) As before, if one has more vector fields on $U$ and on $N-S$, then one has the corresponding formulae for the lower Chern classes.

In 4.2 we can take $M$ to be a neighbouhood of a connected component of the singular set of a holomorphic foliation on some complex manifold. In particular one has: 
4.3 Corollary. Let $\mathcal{F}$ be a p-dimensional holomorphic foliation on a complex $n$ manifold $M, n>p>0$, and let $P$ be an isolated singularity of $\mathcal{F}$. Let $\mathbb{D}$ be a small ball around $P$ in $M$, and suppose there exists on $\mathbb{D}$ a $C^{\infty}$ vector field $v$, singular only at $P$ and tangent to $\mathcal{F}$. Then:

$$
\operatorname{TRes}_{c_{n}}(\mathcal{F}, P)=\tilde{c}_{n}\left(\left.\mathcal{F}\right|_{\mathbb{D}}\right)[\mathbb{D}]+\operatorname{Res}_{\sigma_{n}}(\mathcal{F}, P)
$$

where $\operatorname{Res}_{\sigma_{n}}(\mathcal{F}, P)$ is the corresponding Baum-Bott residue. In particular, if $\mathcal{F}$ is locally free at $P$, then $\operatorname{Res}_{\sigma_{n}}(\mathcal{F}, P)$ is integral and one has:

$$
\operatorname{TRes}_{c_{n}}(\mathcal{F}, P)=\operatorname{Res}_{\sigma_{n}}(\mathcal{F}, P) \text {. }
$$

4.4 Remark. Let $\mathcal{F}$ be a $p$-dimensional holomorphic foliation on a complex $n$ manifold and $Z$ a compact component of the singular set $S$. By [BSu] Theorem (1.2), $\operatorname{Res}_{\sigma_{i}}(\mathcal{F}, Z)$ is an integral class for every $i>n-p$.

\section{$\S 5$. The index of a vector field on a singular variety}

Now let $(V, P)$ be the germ of an isolated complete intersection singularity (ICIS), defined by $k$ holomorphic functions,

$$
f=\left(f_{1}, \ldots, f_{k}\right):\left(U \subset \mathbb{C}^{n+k}, P\right) \rightarrow\left(\mathbb{C}^{k}, 0\right)
$$

and $v$ a continuous vector field on $V$, singular only at $0, n>0$.

5.1 Definition. (The Schwartz index, cf. [Sc,BSc,KT]) If $v$ is everywhere transversal to the link $K$ of $P$ in $V$, its Schwartz index is 1 . Otherwise, let $\tau$ be a vector field on $V$ such that restricted to the link $K=V \cap S_{\varepsilon}$, it is the unit outwards-pointing normal vector field of $K$ in $V$. Let $K^{\prime}=V \cap S_{\delta}$ be another link of $P$ in $V$, with $\delta<\varepsilon$, let $C \subset V-\{P\}$ be the cylinder bounded by $K$ and $K^{\prime}$ and let $X$ be the vector field on $\partial C$ which is $\tau$ on $K$ and $v$ on $K^{\prime}$. The difference between $\tau$ and $v, d(\tau, v) \in \mathbb{Z}$, is the total Poincaré-Hopf index of $X$ in $C$. The Schwartz index of $v$ is:

$$
\operatorname{S-Ind}(v, P)=1+d(\tau, v) \text {. }
$$

The integer $d(\tau, v)$ measures "the lack of radiality" of $v$.

5.2 Definition. (The GSV-index, cf. [Se,GSV,BG,G,SS,LSS]) Suppose $n>1$, or $n=1$ and $V$ is irreducible, so that the link $K$ of $P$ in $V$ is connected. Let $\nabla f_{1}, \ldots, \nabla f_{k}$ be the gradient vector fields of the functions that define $V$. Then $\left(v, \nabla f_{1}, \ldots, \nabla f_{k}\right)$ is a $(k+1)$-frame on all of $U-\{P\}$, which can be made orthonormal by the Gramm-Schmidt process. Hence one has a map

$$
\left(v, \nabla f_{1}, \ldots, \nabla f_{k}\right): K \rightarrow W_{k+1, k+n}
$$


where $W_{k+1, k+n}$ is the Stiefel manifold of complex, orthonormal $(k+1)$-frames in $\mathbb{C}^{n+k}$. The homotopy groups $\pi_{i}\left(W_{k+1, k+n}\right)$ are all zero for $1=0,1, \ldots, 2 n-2$, and $\pi_{2 n-1}\left(W_{k+1, k+n}\right) \simeq \mathbb{Z}$, see $[\mathrm{Hu}]$. Hence the homotopy classes of maps from $K$ into $W_{k+1, k+n}$ are classified by their degree. The GSV-index of $v$ in $V, \operatorname{Ind}(v, P)$, is the degree of the above map $\left(^{*}\right)$.

According to [GSV], $\operatorname{Ind}(v, P)$ is also described as follows. Let $v^{\prime}$ be the vector field on a neighbourhood $N$ of the boundary of a nearby Milnor fibre $F$ obtained by pushing $v$ by a local ambient isotopy carrying a neighbourhood of $K$ in $V$ onto $N$. Then $\operatorname{Ind}(v, P)$ is equal to the total index $\operatorname{Ind}\left(v^{\prime}, F\right)$ of $v^{\prime}$ in $F$. Thus, if $v$ is transversal to $K$, we have

$$
\operatorname{Ind}(v, P)=1+(-1)^{n} \mu
$$

where $\mu$ is the Milnor number of $V$ at $P$. (The above is proved in [GSV] only when $V$ is a hypersurface, but the same proof works in the higher codimensional case as well with $\mu$ defined as in [L].) When $n=1$ and $V$ may not be irreducible, we use this process to define $\operatorname{Ind}(v, P)$, which is also how it is given in [B]. Therefore, in general, one has:

5.3 Proposition. The $S c h w a r t z$ index and the $G S V$-index are related by the formula,

$$
\operatorname{Ind}(v, P)=\mathrm{S}-\operatorname{Ind}(v, P)+(-1)^{n} \mu .
$$

We remark that if $v$ is holomorphic, then its GSV-index (together with the residues for general symmetric homogeneous polynomials of degree $n$ ) at $P \in V$ can be defined via differential geometry [LSS], and if $V$ is a hypersurface, then this index can also be defined via homological algebra [G]. The following theorem follows from 5.3 above and the fact $[\mathrm{BSc}]$ that the Schwartz index gives rise to the top Chern class of a singular variety. We prove it here for completeness.

5.4 Theorem. (Poincaré-Hopf for singular varieties) Let $V$ be a compact, local complete intersection of dimension $n$, with isolated singularities $P_{1}, \ldots, P_{r}$. Let $v$ be a continuous vector field on $V$, which is singular at the $P_{i}$ 's and possibly at some other points $Q_{1}, \ldots, Q_{s}$, which are smooth points of $V$. Let $\operatorname{Ind}(v, V)$ be the sum of the local Poincaré-Hopf indices of $v$ at the $Q_{i}$ 's and the GSV-indices of $v$ at the $P_{i}$ 's. Then,

$$
\operatorname{Ind}(v, V)=\chi(V)+(-1)^{n} \sum_{i=1}^{r} \mu_{i}
$$

independently of $v$, where $\chi(V)$ is the Euler-Poincaré characteristic of $V$ and $\mu_{i}$ is the Milnor number of $V$ at its singular point $P_{i}$.

Proof. Assume first that $v$ is transversal to the link $K_{i}$ of $P_{i}$, for all $P_{i}$ 's. We remove from $V$ small neighbourhoods $B_{1}, \ldots, B_{r}$ of the singular points; Then $V^{*}=V-\cup B_{i}$ 
is a manifold with boundary $\partial V^{*}=K_{1} \cup \cdots \cup K_{r}$, and $v$ is transversal to $\partial V^{*}$. Hence, by the theorem of Poincaré-Hopf for manifolds with boundary [M1], the total index of $v$ in $V^{*}$ is,

$$
\operatorname{Ind}\left(v, V^{*}\right)=\chi\left(V^{*}\right)=\chi(V)-r .
$$

On the other hand, $\operatorname{Ind}\left(v, P_{i}\right)=1+(-1)^{n} \mu_{i}$. Thus one has,

$$
\operatorname{Ind}(v, V)=\chi(V)+(-1)^{n} \sum_{i=1}^{r} \mu_{i}
$$

proving the theorem when $v$ is transversal to $\partial V^{*}$. Now in general, we consider the link $K_{i}$ with the vector field $\left.v\right|_{K_{i}}$; For each $P_{i}$ we take a smaller link $K_{i}^{\prime}$ and we put there a vector field $\tau_{i}^{\prime}$, normal to $K_{i}^{\prime}$ in $V$. So we have a cylinder $C_{i} \simeq K_{i} \times I$ for each $i=1, \ldots, r$, whose boundary is $K \cup K^{\prime}$, and a vector field $\tilde{v}_{i}$ on $\partial C_{i}$, which is $v_{i}$ on $K_{i}$ and $\tau_{i}^{\prime}$ on $K_{i}^{\prime}$. The index of $\tilde{v}_{i}$ in $C_{i}$ is the above "difference" $d\left(\tau_{i}, v_{i}\right)$. One has,

$$
\operatorname{Ind}\left(v, P_{i}\right)=d\left(\tau_{i}, v_{i}\right)+1+(-1)^{n} \mu_{i} .
$$

On the other hand, we can form the manifold $\tilde{V}^{*}$ obtained by attaching to $V^{*}$ the $r$ cylinders $K_{i} \times I$, and we have the vector field $v^{\prime}$ on its boundary $\partial V^{*}=K_{1}^{\prime} \cup \cdots \cup K_{r}^{\prime}$. One has,

$$
\operatorname{Ind}\left(v, V^{*}\right)=\operatorname{Ind}\left(v^{\prime}, \tilde{V}^{*}\right)-\sum_{i=1}^{r} d\left(\tau_{i}, v_{i}\right)
$$

hence,

$$
\begin{aligned}
\operatorname{Ind}(v, V) & =\operatorname{Ind}\left(v, V^{*}\right)+\sum_{i=1}^{r} \operatorname{Ind}\left(v, P_{i}\right) \\
& =\left[\operatorname{Ind}\left(v^{\prime}, \tilde{V}^{*}\right)-\sum_{i=1}^{r} d\left(\tau_{i}, v_{i}\right)\right]+\left[\sum_{i=1}^{r} d\left(\tau_{i}, v_{i}\right)+1+(-1)^{n} \mu_{i}\right] \\
& =\chi\left(\tilde{V}^{*}\right)+\sum_{i=1}^{r}\left[1+(-1)^{n} \mu_{i}\right]=\chi(V)+\sum_{i=1}^{r}(-1)^{n} \mu_{i} .
\end{aligned}
$$

Now we give an application of Theorem 5.4. Assume that $V$ is a local complete intersection in some complex manifold $W$. Thus the normal bundle of its regular part extends (canonically) to a vector bundle $N_{V}$ on $V$. Suppose, furthermore, that $V$ is a "strong" local complete intersection in the sense of [LS], i.e., $N_{V}$ still extends to a $\left(C^{\infty}\right)$ vector bundle on a neighbourhood of $V$ in $W$. This class of varieties include, in particular, every hypersurface with a natural holomorphic extension of $N_{V}$ (the line bundle on $W$ determined by the divisor $V$ ), every complete intersection with a trivial extension of $N_{V}$ and every complete intersection in the projective space $\mathbb{C P}^{n+k}$ with a holomorphic extension of $N_{V}$ depending only on the degrees of polynomials defining $V$. See [LS] for more details. 
5.5 Theorem. For a compact strong local complete intersection $V$ of dimension $n$ with isolated singularities $P_{1}, \ldots, P_{r}$ in $W$, the Chern number $c_{n}\left(\left.T W\right|_{V}-N_{V}\right)[V]$ of its virtual tangent bundle $\left.T W\right|_{V}-N_{V}$ is given by

$$
c_{n}\left(\left.T W\right|_{V}-N_{V}\right)[V]=\chi(V)+(-1)^{n} \sum_{i=1}^{r} \mu_{i}
$$

This is a consequence of Theorem 5.4 and the following two lemmas.

5.6 Lemma. Let $V$ be a complex analytic subvariety of dimension $n>0$ with isolated singularities $P_{1}, \ldots, P_{r}$ in a complex manifold $W$. Then there exists a $C^{\infty}$ vector field $v$ on $V$, singular at the $P_{i}$ 's and at a (possibly empty) finite set of other points.

Proof. It follows from [M2] that there is a $C^{\infty}$ vector field $X_{i}$ on a neighbourhood $B_{i}$ of each $P_{i}$ in $W$, which is singular only at $P_{i}$ and is tangent to $V$. Let $D_{i}=B_{i} \cap V$ and let $v_{i}$ be the restriction of $X_{i}$ to $D_{i}$. Then $V^{*}=V-\cup_{i=1}^{r} \operatorname{Int}\left(D_{i}\right)$ is a smooth manifold with boundary, and the $v_{i}$ 's determine a non-singular vector field on the boundary of $V^{*}$. By elementary obstruction theory [St], this can be extended to a $C^{\infty}$ vector field on all of $V^{*}$, with at most a finite number of singularities.

Let $V$ be a strong local complete intersection in $W$ and $v$ a $C^{\infty}$ vector field on $V$ with singular set $S$ (which contains the singular set of $V$ ). For each compact component $Z$ of $S$, we may define the virtual index $\mathrm{v}-\operatorname{Ind}(v, Z)$ of $v$ at $Z$ (see the proof of Lemma 5 in [LSS], where it is denoted by $\mathrm{v}-\operatorname{Ind}_{Z}(v)$ ) so that, if $V$ is compact, we have

$$
\sum_{Z \subset S} \mathrm{v}-\operatorname{Ind}(v, Z)=c_{n}\left(\left.T W\right|_{V}-N_{V}\right)[V]
$$

Note that if $Z$ is in the regular part of $V, \mathrm{v}-\operatorname{Ind}(v, Z)$ coincides with the total index of $v$ in a small neighbourhood of $Z$.

The following lemma is proved as [LSS] Lemma 5, noting that at the final stage of its proof, the vector fields $\tilde{X}$ and $X$ may only be $C^{\infty}$ and that the holomorphic tangent and normal bundles are naturally identified with the corresponding real bundles.

5.7 Lemma. Let $P$ be an isolated singular point of a strong local complete intersection $V$ in $W$ and let $X$ be a $C^{\infty}$ vector field in a neighbourhood of $P$ in $W$, which is singular only at $P$ and is tangent to $V$. Then the virtual index at $P$ of the restriction $v$ of $X$ to $V$ coincides with its $G S V$-index;

$$
\mathrm{v}-\operatorname{Ind}(v, P)=\operatorname{Ind}(v, P)
$$


5.8 Remarks. 1. Suppose $\operatorname{dim} W=2$ and $\operatorname{dim} V=n=1$. If $V$ is compact, from the "adjunction formula" ([Ko] (2.2)), we have

$$
-\chi(\tilde{V})=\left(K_{W}+V\right) \cdot V-\sum_{i=1}^{r} c_{P_{i}}(V) .
$$

Here $\tilde{V}$ is a non-singular model of $V, K_{W}$ is the canonical divisor of $W$ and $c_{P_{i}}(V)$ is an invariant of $V$ at the singular point $P_{i}$, which is related to the Milnor number $\mu_{i}$ by $c_{p_{i}}(V)=\mu_{i}+s_{i}-1$ with $s_{i}$ the number of (local) branches of $V$ at $P_{i}$. Since $\chi(\tilde{V})-\sum_{i=1}^{r}\left(s_{i}-1\right)=\chi(V)$ and $\left(K_{W}+V\right) \cdot V=-c_{1}\left(\left.T W\right|_{V}-N_{V}\right)[V]$, we see that the formula in 5.5 is equivalent to the above formula.

2. If $V$ is a complete intersection in $W=\mathbb{C P}^{n+k}, N_{V}$ is determined by its multidegree $\left(d_{1}, \ldots, d_{k}\right)$ and we have

$$
c_{n}\left(\left.T W\right|_{V}-N_{V}\right)[V]=\left[(1+h)^{n+k+1} \cdot \prod_{i=1}^{k} \frac{d_{i}}{1+d_{i} h}\right]_{n}
$$

where $h$ denotes the first Chern class of the hyperplane bundle and [ $]_{n}$ the coefficient of $h^{n}$ in [ ]. In particular, for a hypersurface $V$ of degree $d$, we have, from Theorem 5.5,

$$
\chi(V)=\frac{1}{d}\left((1-d)^{n+2}+(n+2) d-1\right)+(-1)^{n+1} \sum_{i=1}^{r} \mu_{i}
$$

Also, for a complete intersection $V, 5.5$ implies the following formula, which is readily proved by a direct argument as well (cf. [D] Ch.5, Corollary (4.4)):

$$
\chi(V)=\chi\left(V_{0}\right)+(-1)^{n+1} \sum_{i=1}^{r} \mu_{i}
$$

where $V_{0}$ is a non-singular complete intersection in $\mathbb{C P}^{n+k}$ of dimension $n$ with the same multidegree as $V$.

3. In $[P]$, a generalized Milnor number is defined for each compact connected component of the singular set of a hypersurace and a formula for the sum of these numbers is proved ([P] Proposition 1.6). The formula, which is given under the assumtion that the ambient space be compact, coincides with the one in Theorem 5.5 , if the singularities are isolated. 


\section{§6. The index for holomorphic foliations on singular varieties}

Let $(V, P)$ be an ISIC as before. We now consider a field $\mathcal{D}$ of complex $p$ planes on $V$, singular only at $P$. Let $\mathcal{D}^{\perp}$ be the normal bundle of $\mathcal{D}$ in $V^{*}=V-\{P\}$, with respect to some riemanian metric, so $\mathcal{D}^{\perp}$ is a field of complex $(n-p)$-planes. We let $K$ be the link of $P$ in $V$.

6.1 Lemma. Let $v$ and $s$ be continuous, nowhere zero vector fields on $V$. Assume further that $p \neq \frac{n}{2}, \frac{n+1}{2}$ or else that the link $K$ is a homology sphere. If $v$ and $s$ are both contained in $\mathcal{D}$, then $v$ and $s$ have the same local GSV-index at $P$,

$$
\operatorname{Ind}(v, P)=\operatorname{Ind}(s, P)
$$

Proof. Let $K_{\varepsilon}$ and $K_{\delta}$ be small links of $P$ in $V, \delta<\varepsilon$, and let $C$ be the cylinder bounded by $K_{\varepsilon}$ and $K_{\delta}$. Let $Z$ be the vector field on $\partial C$ given by $v$ on $K_{\varepsilon}$ and $s$ on $K_{\delta}$. The tangent bundle of $C$ splits as the direct sum of $\left.\mathcal{D}\right|_{C}$ and $\left.\mathcal{D}^{\perp}\right|_{C}$. Hence one has,

$$
e(T C, Z)=e\left(\left.\mathcal{D}\right|_{C}, Z\right) \cdot e\left(\left.\mathcal{D}^{\perp}\right|_{C}\right)
$$

where

$$
e\left(\left.\mathcal{D}\right|_{C}, Z\right) \in H^{2 p}(C, \partial C) \simeq H_{2 n-2 p}(C) \simeq H_{2 n-2 p}(K),
$$

is the Euler class of $\left.\mathcal{D}\right|_{C}$ relative to $Z$ and,

$$
e\left(\left.\mathcal{D}^{\perp}\right|_{C}\right) \in H^{2 n-2 p}(C) \simeq H^{2 n-2 p}(K) \simeq H_{2 p-1}(K)
$$

is the usual Euler class of $\left.\mathcal{D}^{\perp}\right|_{C}$. The homology groups $H_{i}(K)$ are well understood $[\mathrm{M} 2, \mathrm{Ha}]$, they are all 0 except (possibly) for $i=0, n-1, n$. Thus, if $2 p \neq n, n+1$, or if $K$ is a homology sphere, then $e(T C, Z)=0$ and 6.1 follows.

6.2 Definition. Let $P$ be an isolated singularity of a field $\mathcal{D}$ of $p$-planes on $V$, with $2 p \neq n, n+1$. Assume that on a neighbourhood of $\mathcal{D}$ in $V$, there exists a nowhere zero vector field $v$ contained in $\mathcal{D}$. The local GSV-index of $\mathcal{D}$ at $P$, $\operatorname{Ind}(\mathcal{D}, P)$, is the GSV-index of $v$ at $P$. If $\mathcal{F}$ is a holomorphic foliation on $V$, singular only at $P$, then its local GSV-index is the index of $\mathcal{D}=T \mathcal{F}$, the tangent bundle of $\mathcal{F}$.

We remark that the index of $\mathcal{D}$ is not always defined: We need to have a vector field $v$ as above, but if there exists one such $v$, then the index of $\mathcal{D}$ does not depend on the choice of $v$, by 6.1. This condition is always satisfied for foliations given by the action of a (complex) Lie group, or more generally, for foliations which are free at $P$. We note that if $P$ is a regular point of $V$, then the local index of $\mathcal{D}$ is the topological Euler residue of $\S 2$ above.

6.3 Definition. Let $(V, P)$ and $\left(V^{\prime}, P\right)$ be $n$-dimensional ICIS in $\mathbb{C}^{n+k}$, defined by holomorphic functions

$$
f_{1}, f_{2}:\left(U \subset \mathbb{C}^{n+k}, P\right) \rightarrow\left(\mathbb{C}^{k}, 0\right),
$$


on an open set $U$ of $\mathbb{C}^{n+k}$. We say that the germs at $P$ of $f_{1}$ and $f_{2}$ are topologically equivalent if there exists an orientation preserving local homeomorphism $h$ of $\mathcal{U}$ around 0 such that

$$
f_{2}=f_{1} \circ h \text {. }
$$

$h$ is called a topological equivalence between these two germs.

6.4 Definition. Let $(V, P)$ and $\left(V^{\prime}, P\right)$ be $n$-dimensional ICIS in $\mathbb{C}^{n+k}$ and let $\mathcal{F}$ and $\mathcal{F}^{\prime}$ be $p$-dimensional holomorphic foliations on $V$ and $V^{\prime}$, respectively, singular only at $P$. These two foliations are topologically equivalent at $P$ if there exists a topological equivalence $h$ between $(V, P)$ and $\left(V^{\prime}, P\right)$ taking the leaves of $\mathcal{F}$ onto the leaves of $\mathcal{F}^{\prime}$.

6.5 Theorem. The local GSV-index of a holomorphic foliation $\mathcal{F}$ on an ICIS $(V, P)$ is a topological invariant. This is, if $\mathcal{F}^{\prime}$ is a holomorphic foliation on an ICIS $\left(V^{\prime}, P\right)$ and $\mathcal{F}^{\prime}$ is topologically equivalent to $\mathcal{F}$, then:

(i) The GSV-index of $\mathcal{F}$ at $P$ is defined if and only if the GSV-index of $\mathcal{F}^{\prime}$ is defined.

(ii) If these indices are defined, then one has:

$$
\operatorname{Ind}(\mathcal{F}, P)=\operatorname{Ind}\left(\mathcal{F}^{\prime}, P\right) .
$$

The proof of this theorem is analogous to the proof of theorem 4.5 in [GSV]. The idea is to state the problem in the appropriate category: A homeomorphism does not carry a vector field onto a vector field, but it does carry a flow onto a flow; So we discuss first how the concept of index extends to flows [GSV]:

6.6 Definition. Let $\left\{\varphi_{t}\right\}, t \in \mathbb{R}$, be a continuous flow on an open set $U \subset \mathbb{R}^{m}$, $m>1$, and assume $0 \in U$ is an isolated stationary point of $\left\{\varphi_{t}\right\}$, i.e., $\varphi_{t}(0)=0$ for all $t$ and there exists a neighbourhood $N$ of 0 and a time $s_{0}>0$ such that $\varphi_{t}(x) \neq x$ for every $x \in N-\{0\}$ and $t \in\left(0, s_{0}\right)$. Let $S_{\varepsilon} \in N$ be a sphere of radius $\varepsilon$ and centered at 0 , for some fixed $\varepsilon>0$. Then the index of $\left\{\varphi_{t}\right\}$, denoted $\operatorname{Ind}\left\{\varphi_{t}\right\}$, is the degree of the map

$$
\Phi_{t, \varepsilon}(x)=\frac{\varphi_{t}(x)-x}{\left\|\varphi_{t}(x)-x\right\|}: S_{\varepsilon} \rightarrow S^{m-1} \subset \mathbb{R}^{m},
$$

for some fixed $t \in\left(0, s_{0}\right)$.

It is shown in [GSV] that this definition does not depend on the choice of $\varepsilon$ nor $t$, and the sphere $S_{\varepsilon}$ can be taken to be any topological $(m-1)$-sphere embedded in $N$ containing 0 in the bounded component of its complement. If the flow $\left\{\varphi_{t}\right\}$ is differentiable, then its index equals that of its tangent vector field.

We now let $\left\{\varphi_{t}\right\}$ be a continuous flow on an ICIS $(V, P)$ with an isolated stationary point at $P$. We construct a vector field $X$ on the link $K$ of $P$ in $V$ by joining each $x \in K$ with the point $\varphi_{t}(x)$, and projecting it orthogonally to a tangent vector field $X$ of $V$ over $K$. The index of $\left\{\varphi_{t}\right\}$, denoted $\operatorname{Ind}\left(\left\{\varphi_{t}\right\}, P\right)$, is the index of $X$. The proof of the following lemma is a mimic of the proof of Lemma 6.1 above, so we leave it to the reader: 
6.7 Lemma. Let $\mathcal{F}$ be a holomorphic foliation on $(V, P)$, and let $\left\{\varphi_{t}\right\}$ be a continuous flow on $(V, P)$ with an isolated stationary point at $P$, for which the leaves of $\mathcal{F}$ are invariant sets. Then

$$
\operatorname{Ind}\left(\left\{\varphi_{t}\right\}, P\right)=\operatorname{Ind}(\mathcal{F}, P) .
$$

Let us now prove Theorem 6.5: Assume the index of $\mathcal{F}$ at $P$ is defined, so one has a non-singular vector field $X$ on $V-\{P\}$, tangent to $\mathcal{F}$. Let $\left\{\varphi_{t}\right\}$ be the flow of $X$ and define a flow $\left\{\psi_{t}\right\}$ on $V^{\prime}$ by $\left\{\psi_{t}\right\}=\left\{h \varphi_{t} h^{-1}\right\}$, where $h$ is a topological equivqlence between $\mathcal{F}$ and $\mathcal{F}^{\prime}$. Then the orbits of $\left\{\psi_{t}\right\}$ are contained in the leaves of $\mathcal{F}^{\prime}$ and $\left\{\psi_{t}\right\}$ has a single stationary point at $P$, hence the index of $\mathcal{F}^{\prime}$ at $P$ is defined. Let us now prove that the indices of $\mathcal{F}$ and $\mathcal{F}^{\prime}$ coincide. for this we move the vector field $X$ by an isotopy to obtain a non-singular vector field on a neighbourhood of the link $K$ on a Milnor fibre $F$ of $V$. We extend $X$ to a vector field on all of $F$ with isolated singularities (which is always possible by elementary obstruction theory). The sum of all the local indices of $X$ on $F$ equals the index of $\mathcal{F}$. Now, the homeomorphism $h$ carries the flow $\left\{\varphi_{t}\right\}$ of $X$ on $F$ to a continuous flow $\left\{\psi_{t}\right\}=\left\{h \varphi_{t} h^{-1}\right\}$ on a Milnor fibre $F^{\prime}$ of $V^{\prime}$; The singularities of $X$ on $F$ go to singularities of $\left\{\psi_{t}\right\}$ on $F^{\prime}$, and the sum of the local indices of $\left\{\psi_{t}\right\}$ is the index of $\mathcal{F}^{\prime}$. Thus we only have to prove that the local index of $\left\{\varphi_{t}\right\}$ at a stationary point $Q_{i}$ equals the local index of $\left\{\psi_{t}\right\}$ at the stationary point $h\left(Q_{i}\right)$, but this is lemma 4.2 in [GSV].

Since the local index of a field of planes $\mathcal{D}$ at a singular point $P$ of $V$ is actually given by the local index of a vector field, all the formulae of [GSV, BG, G, SS, LSS] apply for the index of $\mathcal{D}$. In particular, let $(V, P)$ be an ICIS of dimension $n$, let $\mathcal{F}$ be a $p$-dimensional holomorphic foliation on $V-\{P\}$, and let $v$ be a continuous vector field on $V$, singular only at $P$ and tangent to $\mathcal{F}$. Let $\pi: \tilde{V} \rightarrow V$ be a resolution of $P$, let $\tilde{\mathcal{F}}$ be the strict transform of $\mathcal{F}$ in $\tilde{V}$, and let $\tilde{X}$ be the lifting of $v$ to $\tilde{V}^{*}=\tilde{V}-\pi^{-1}(P)$. The following theorem, which is a generalization of Theorem III in [SS], is a consequence of Theorem II of [SS] together with Theorem 1.4 above.

6.8 Theorem. Let $n=2 k$ be even, and let $q_{n}=\frac{(-1)^{k-1} \mathcal{B}_{k}}{2 k !}$, where $\mathcal{B}_{k}$ is the $k$-th Bernoulli number, be the coefficient of $c_{n}$ in the $n$-th Todd polynomial, see [Hi]. Suppose $p \neq k$ and $\tilde{\mathcal{F}}$ is locally free. Then:

$$
\begin{aligned}
\operatorname{Ind}(\mathcal{F}, P)= & c_{p}(\tilde{\mathcal{F}}, \tilde{X}) \cdot c_{n-p}(Q)[\tilde{V}]+\sum_{Z \subset S} \sum_{i=n-p+1}^{n} c_{n-i}(\tilde{\mathcal{F}}) \frown \operatorname{Res}_{\sigma_{i}}(\tilde{\mathcal{F}}, Z) \\
& +\frac{1}{q_{n}}\left(T d^{*}[\tilde{V}]+p_{g}\right)
\end{aligned}
$$

where the sum runs over the connected components of the singular set $S$ of $\tilde{\mathcal{F}}$, $\operatorname{Res}_{\sigma_{i}}(\tilde{\mathcal{F}}, Z)$ are the corresponding Baum-Bott residues, $Q$ is the normal sheaf of 
$\tilde{\mathcal{F}}, p_{g}$ is the geometric genus of $P$, and $T d^{*}[\tilde{V}]$ is the $n$-th Todd polynomial in the relative Chern numbers of $\tilde{V}$ relative to some (any) trivialization of $\left.T \tilde{V}\right|_{\tilde{V}^{*}}$, but taking $c_{n}=0$. In particular, if some component of $S$ consists of an isolated point $P_{0}$, then

$$
\operatorname{Res}_{\sigma_{i}}\left(\tilde{\mathcal{F}}, P_{0}\right)= \begin{cases}0, & \text { for } i=n-p+1, \ldots, n-1 \\ \operatorname{Ind}\left(\tilde{\mathcal{F}}, P_{0}\right), & \text { for } i=n\end{cases}
$$

For instance, if $n=2$ then $q_{n}=\frac{1}{12}$ and $q_{n} \cdot T d^{*}[\tilde{V}]$ is $K^{2}$, the self-intersection number of the canonical class of $\tilde{V}$. Of course this formula is also valid when $V$ is regular at $P$ and $\tilde{V}$ is the result of performing finitely many blow-ups over $P$; in this case the formula is a little simpler because the genus $p_{g}$ is zero. Note that, without the assumption $p \neq k$, the right hand side of the equality in 6.8 gives $\operatorname{Ind}(v, P)$, the GSV-index of $v$ at $P$. If $\tilde{\mathcal{F}}$ is not locally free in 6.8 , then the sum on the right must also include the products of the higher classes of $\tilde{\mathcal{F}}, c_{i}(\tilde{\mathcal{F}}), i>p$, by classes of $Q$. We also note that the right hand side of 6.8 can be expressed in terms of the above "topological residues" using Theorem 4.2.

\section{REFERENCES}

[AH] M.F. Atiyah and F. Hirzebruch, Analytic cycles on complex manifolds, Topology 1 (1961), 25-45.

[BB] P. Baum and R. Bott, Singularities of holomorphic foliations, J. Differential Geom. 7 (1972), 279-342.

[BG] C. Bonatti and X. Gómez-Mont, The index of holomorphic vector fields on singular varieties I, Complex Analytic Methods in Dynamical Systems, IMPA 1992, Astérisque 222, Société Mathématique de France, 1994, pp. 9-35.

[BSc] J.-P. Brasselet et M.-H. Schwartz, Sur les classes de Chern d'un ensemble analytique complexe, Caractéristique d'Euler-Poincaré, Astérisque 82-83, Société Mathématique de France, 1981, pp. 93-147.

[BSu] J.-P. Brasselet and T. Suwa, Nash residues of singular holomorphic foliations, to appear in the Proceedings of the Symposium on Singularity of Hypersurfaces, Fundamental Groups and Finite Covering Maps, Tokyo Institute of Technology, October 1995.

[B] M. Brunella, Feuilletages holomorphes sur les surfaces complexes compactes, preprint.

[CLS] C. Camacho, A. Lins Neto and P.Sad, Topological invariants and equidesingularization for holomorphic vector fields, J. Differential Geom. 20 (1984), 143-174.

[D] A. Dimca, Singularities and Topology of Hypersurfaces, Springer-Verlag, New York, Heidelberg, Berlin, 1992.

[G] X. Gómez-Mont, An algebraic formula for the index of a vector field on a variety with an isolated singularity, preprint.

[GSV] X. Gómez-Mont, J. Seade and A. Verjovsky, The index of a holomorphic flow with an isolated singularity, Math. Ann. 291 (1991), 737-751.

[Ha] H. Hamm, Lokale topologische Eigenschaften komplexer Räume, Math. Ann. 191 (1971), 235-252.

[He] J. Heitsch, $A$ cohomology for foliated manifolds, Comment. Math. Helv. 50 (1975), 197-218.

[Hi] F. Hirzebruch, Topological Methods in Algebraic Geometry, Springer-Verlag, New York, Heidelberg, Berlin, 1966. 
[Hu] D. Husemoller, Fibre bundles, Springer-Verlag, New York, Heidelberg, Berlin, 1975.

[Ke] M. Kervaire, Relative characteristic classes, Am. J. Math. 79 (1957), 517-558.

[KT] H. King and D. Trotman, Poincaré-Hopf theorems on stratified sets, Prépublication Univ. de Provence 1994.

[Ko] K. Kodaira, On compact complex analytic surfaces, I, Ann. of Math. 71 (1960), 111-152.

[L] E. Looijenga, Isolated Singular Points on Complete Intersections, London Mathematical Society Lecture Note Series 77, Cambridge Univ. Press, Cambridge, London, New York, New Rochelle, Melbourne, Sydney, 1984.

[LSS] D. Lehmann, M. Soares and T. Suwa, On the index of a holomorphic vector field tangent to a singular variety, Bol. Soc. Bras. Mat. 26 (1995), 183-199.

[LS] D. Lehmann and T. Suwa, Residues of holomorphic vector fields relative to singular invariant subvarieties, J. Differential Geom. 42 (1995), 165-192.

[M1] J. Milnor, Topology from the Differentiable Viewpoint, Univ. Press of Virginia, Charlottesville, 1965.

[M2] J. Milnor, Singular Points of Complex Hypersurfaces, Annales of Mathematics Studies 61, Princeton University Press, Princeton, 1968.

[MS] J. Milnor and J. Stasheff, Characteristic Classes, Annales of Mathematics Studies 76, Princeton University Press, Princeton, 1974.

[P] A. Parusiński, A generalization of the Milnor number, Math. Ann. 281 (1988), 247-254.

[Sc] M.-H. Schwartz, Classes caractéristiques définies par une stratification d'une variété analytique complexe, CRAS 260 (1965), 3262-3264, 3535-3537.

[SW] P. Schweitzer and A. Whitman, Pontryagin polynomial residues of isolated foliation singularities, Differential Topology, Foliations and Gelfand-Fuks Cohomology, Lecture Notes in Mathematics 652, Springer-Verlag, New York, Heidelberg, Berlin, 1978, pp. 95-103.

[Se] J.A. Seade, The index of a vector field on a complex surface with singularities, The Lefschetz Centennial Conf., ed. A. Verjovsky, Contemp. Math. 58 Part III, Amer. Math. Soc., Providence, 1987, pp. 225-232.

[SS] J.A. Seade and T. Suwa, A residue formula for the index of a holomorphic flow, to appear in Math. Ann..

[St] N. Steenrod, The Topology of Fibre Bundles, Princeton Univ. Press, Princeton, 1951.

[Su1] T. Suwa, Residues of complex analytic foliation singularities, J. Math. Soc. Japan 36 (1984), 37-45.

[Su2] T. Suwa, Chern classes of a local complete intersection, in preparation.

[T] E. Thomas, Fields of tangent 2-planes on even dimensional manifolds, Ann. of Math. 86 (1967), 334-347.

Instituto de Matemáticas, Universidad Nacional Autónoma de México, CiuDad Universitaria, Circuito Exterior, MÉxico 04510 D.F., MÉxico

E-mail address: jseade@gauss.matem.unam.mx

Department of Mathematics, Hokkaido University, Sapporo 060, Japan

E-mail address: suwa@math.hokudai.ac.jp 